\title{
The Sun at millimeter wavelengths
}

\section{Small-scale dynamic events in ALMA Band 3}

\author{
Henrik Eklund ${ }^{1,2}$, Sven Wedemeyer ${ }^{1,2}$, Mikolaj Szydlarski ${ }^{1,2}$, \\ Shahin Jafarzadeh ${ }^{1,2}$, and Juan Camilo Guevara Gómez ${ }^{1,2}$ \\ 1 Rosseland Centre for Solar Physics, University of Oslo, Postboks 1029 Blindern, 0315 Oslo, Norway \\ e-mail: henrik.eklund@astro.uio.no \\ 2 Institute of Theoretical Astrophysics, University of Oslo, Postboks 1029 Blindern, 0315 Oslo, Norway
}

Received 23 April 2020 / Accepted 9 October 2020

\begin{abstract}
Context. Solar observations with the Atacama Large Millimeter/sub-millimeter Array (ALMA) facilitate studies of the atmosphere of the Sun at chromospheric heights at high spatial and temporal resolution at millimeter wavelengths.

Aims. ALMA intensity data at millimeter(mm)-wavelengths are used for a first detailed systematic assessment of the occurrence and properties of small-scale dynamical features in the quiet Sun.

Methods. We analyzed ALMA Band 3 data $(\sim 3 \mathrm{~mm} / 100 \mathrm{GHz})$ with a spatial resolution of $\sim 1.4-2.1$ arcsec and a duration of $\sim 40 \mathrm{~min}$ together with SDO/HMI magnetograms. The temporal evolution of the $\mathrm{mm}$ maps is studied to detect pronounced dynamical features, which then are connected to dynamical events via a $k$-means clustering algorithm. We studied the physical properties of the resulting events and explored whether or not they show properties consistent with propagating shock waves. For this purpose, we calculated observable shock wave signatures at $\mathrm{mm}$ wavelengths from one- and three-dimensional model atmospheres.

Results. We detect 552 dynamical events with an excess in brightness temperature $\left(\Delta T_{\mathrm{b}}\right)$ of at least $\geq 400 \mathrm{~K}$. The events show a large variety in size up to $\sim 9^{\prime \prime}$, amplitude $\Delta T_{\mathrm{b}}$ up to $\sim 1200 \mathrm{~K}$ with typical values in the range $\sim 450-750 \mathrm{~K}$, and lifetime at full width at half maximum of $\Delta T_{\mathrm{b}}$ of between $\sim 43$ and $360 \mathrm{~s}$, with typical values between $\sim 55$ and $125 \mathrm{~s}$. Furthermore, many of the events show signature properties suggesting that they are likely produced by propagating shock waves.

Conclusions. There are a lot of small-scale dynamic structures detected in the Band 3 data, even though the spatial resolution sets limitations on the size of events that can be detected. The number of dynamic signatures in the ALMA mm data is very low in areas with photospheric footpoints with stronger magnetic fields, which is consistent with the expectation for propagating shock waves.
\end{abstract}

Key words. Sun: chromosphere - Sun: radio radiation - Sun: atmosphere - Sun: magnetic fields - shock waves techniques: interferometric

\section{Introduction}

The Atacama Large Millimeter/sub-millimeter Array (ALMA) provides new diagnostic possibilities for studying the dynamical nature of the solar chromosphere at millimeter $(\mathrm{mm})$ wavelengths thanks to its high spatial and temporal resolution. The mm-wavelength radiation observable with ALMA originates at chromospheric heights (Wedemeyer et al. 2016, and references therein) from free-free continua emission under conditions of local thermodynamic equilibrium (LTE). The measured continuum intensities (and equivalently the continuum brightness temperatures) are therefore expected to depend linearly on the local thermal gas temperature, or more precisely, the electron temperature.

The solar chromosphere is a highly dynamic and structured region of the Sun's atmosphere that features a variety of phenomena on different spatial scales. In particular, small-scale structures have been shown to evolve often on short timescales on the order of a few seconds or less (Okamoto \& De Pontieu 2011; Morton 2012; Gafeira et al. 2017; Jafarzadeh et al. 2017a,b). Therefore, both high spatial and temporal resolution are needed to fully understand this dynamic region. It is also worth nothing that most of the commonly employed chromospheric diagnostics (at ultraviolet, optical, and infrared wavelengths) are formed under non-LTE conditions (de la Cruz Rodríguez \& van Noort
2017; Carlsson et al. 2019), which as a result, renders the meaningful interpretation of such observations challenging. Thus, by promising to provide direct measurements of temperatures under LTE conditions at high temporal resolution, ALMA is an excellent tool for studying dynamic small-scale structures in the solar chromosphere (see, e.g., Guevara Gómez et al. 2020).

Observations of the quiet-Sun internetwork chromosphere in the Ca II K line revealed a bright mesh-like pattern of elongated structures with fainter intermediate areas outside strong magnetic field concentrations (Wöger et al. 2006). The typical mesh size of the observed pattern, which is produced by the interaction of propagating shock waves, was determined to be on the order of $1^{\prime \prime} .95$. Numerical simulations of the solar atmosphere predict that a corresponding pattern should be observable with ALMA (Wedemeyer-Böhm et al. 2007; Loukitcheva et al. 2015; Wedemeyer et al. 2016, and references therein).

The corresponding shock wave signatures at $\mathrm{mm}$ wavelengths as observable with ALMA have been predicted by Loukitcheva et al. (2004, 2006) using one-dimensional models and by Eklund et al. (2020) using a three-dimensional model (cf. Wedemeyer-Böhm et al. 2007). These latter authors found large brightness temperature variations with amplitudes of thousands of Kelvin. Studies of observational ALMA data on the other hand indicate smaller temperature variations on the order of several hundred Kelvin (see, e.g. Patsourakos et al. 2020; 
Nindos et al. 2018; Alissandrakis et al. 2017; Wedemeyer et al. 2020; Loukitcheva et al. 2019).

We note that the spatial resolution of available ALMA band 3 data used in publications is around 2 " or below (e.g., Loukitcheva et al. 2019) with the minor beam axis down to $1.4^{\prime \prime}$ (see Wedemeyer et al. 2020, and references therein) and is therefore on the same order as the aforementioned typical spatial scale of the shock-wave-induced mesh-like pattern. The visibility of this pattern and with it the amplitude of observed brightness temperature variations therefore critically depend on the angular resolution achieved with ALMA. At the same time, ALMA provides a very useful tool to study and understand shock waves and the general dynamics of the chromosphere given that the measured brightness temperatures are expected to be closely related to the local electron temperature in the probed layer (Wedemeyer et al. 2016, and references therein). Accurate statistics of the spatial and temporal scales as well as the amplitudes of the brightness temperature variations can also be used as feedback to numerical models of the solar atmosphere in order to refine simulations, making them even more accurate and realistic.

Here, we analyze one of the first regular Band $3(\sim 3 \mathrm{~mm} /$ $100 \mathrm{GHz}$ ) observations of the Sun with ALMA (Cycle 4), which has been described in Wedemeyer et al. (2020). This high cadence $(2 \mathrm{~s})$ time series is used for a statistical study of detected brightness temperature signatures of small-scale dynamic events. An important question in this regard pertains to whether or not the detected signatures are caused by propagating shock waves in the solar chromosphere. This paper is structured in the following way: in Sect. 2, the properties and the processing of the observational data are described. In Sect. 3, the statistics of the detected dynamical features are presented. In Sect. 4 we further discuss the results and, with the support of numerical simulations, investigate the possibility that the detected events are signatures of propagating shock waves. In that respect, the limitations of the observational data are addressed. In Sect. 5, we summarize and conclude the results and offer a brief outlook on future work.

\section{Methods}

\subsection{Observational data}

The observational data studied in this paper were obtained with ALMA in receiver band 3 on December 22, 2016 (project ID: 2016.1.00423.S), targeting a quiet Sun region with a few magnetic network elements close to solar disk center. Observations were made between 14:19 UT and 15:07 UT separated into four time scans in blocks of $10 \mathrm{~min}$ each, separated by calibration gaps of $\sim 2.5 \mathrm{~min}$. The data set was processed using the Solar Alma Pipeline (SoAP). The specifics of SoAP will be explained in detail in a forthcoming paper (Szydlarski et al., in prep., see also Wedemeyer et al. 2020). In summary, the data set was calibrated (with a script delivered together with the data from the ALMA Science Archive) followed by deconvolution using the Multiscale CLEAN algorithm (Cornwell 2008) and primary beam correction, which results in interferometric intensity maps. These are combined ("feathered") with single-dish total power measurements. The intensities were transformed into brightness temperatures under the assumption of the Rayleigh-Jeans law and formation in LTE (see, e.g., Wilson et al. 2013) through the relation

$T_{\mathrm{b}}=\frac{c^{2} I_{v}}{2 k_{\mathrm{B}} v^{2}}$
Table 1. Span of wavelength and frequency of the sub-bands of ALMA spectral band 3 .

\begin{tabular}{lccccccc}
\hline \hline \multicolumn{7}{c}{ Band 3 } \\
\cline { 1 - 2 } Sub-band & Min & Mid & Max & & Min & Mid & Max \\
\cline { 2 - 3 } \cline { 7 - 9 } SB1 & 3.189 & 3.224 & 3.259 & & 92.0 & 93.0 & 94.0 \\
SB2 & 3.123 & 3.156 & 3.189 & & 94.0 & 95.0 & 96.0 \\
SB3 & 2.828 & 2.856 & 2.883 & & 104.0 & 105.0 & 106.0 \\
SB4 & 2.776 & 2.802 & 2.828 & & 106.0 & 107.0 & 108.0 \\
\hline
\end{tabular}

where $c$ is the speed of light and $k_{\mathrm{B}}$ is the Boltzmann constant. The images were then combined into a time series. The final dataset has a cadence of $2 \mathrm{~s}$, with a total of 1200 timesteps. For an in-depth analysis and details on this specific dataset, see Wedemeyer et al. (2020), where the quality and limitations of the data are evaluated. The same dataset is analyzed in Jafarzadeh et al. (2020), where global oscillations in the time-series of images (along with other ALMA datasets) are studied.

For solar observations, ALMA receiver band 3 is currently set up with four spectral sub-bands as indicated in Table 1. The formation height of the mm-wavelength radiation is wavelength dependent. ALMA band 3 is thought to be formed in the upper chromosphere (Wedemeyer et al. 2020; White et al. 2017, and references therein). The sub-bands are grouped pairwise where SB1 is adjacent to SB2, and SB3 is adjacent to SB4. Differences between the sub-bands can in principle be used to study the small-scale structure along the line of sight (see e.g., Jafarzadeh et al. 2019; Rodger et al. 2019) although simulations imply that the differences are typically small (see, e.g., Eklund et al. 2020). However, in the present work, we choose to combine all spectral channels of all four sub-bands in the deconvolution process when producing the intensity maps as this results in better sampling of the Fourier space and thus a higher signal-to-noise ratio, which is advantageous for the detection and analysis of small-scale dynamic features.

The output of the image reconstruction is cut off at a radius of $32.8^{\prime \prime}$ from the center field of view (FOV). The cut-off point corresponds to 0.3 times the main beam power response. The pixel size of the final images is 0.32 ". The "clean" beam, that is, a Gaussian fit to the main lobe of the point spread function (PSF) of the interferometric array constitutes the resolution element. The full width at half maximum (FWHM) of the clean beam varies slightly over time but has a mean value of about $1.4^{\prime \prime}$ and $2.1^{\prime \prime}$ along the minor and major axis, respectively, with a beam position angle of $68^{\circ}$.

Magnetic-field measurements are taken with the Helioseismic Magnetic Imager (HMI; Schou et al. 2012) on board the Solar Dynamics Observatory (SDO; Pesnell et al. 2012). The magnetograms, measuring the Fe I line (6173 $\AA$ ) at photospheric heights, have a cadence of $36 \mathrm{~s}$ and are co-aligned with the ALMA observations. The SDO images are re-sampled to a pixel size of $0.32^{\prime \prime}$ to match the ALMA data. For the spatial co-alignment, the ALMA images were cross-correlated with a combination of the $170 \mathrm{~nm}$ and $30.4 \mathrm{~nm}$ channels of the SDO's Atmospheric Imaging Assembly (AIA; Lemen et al. 2012).

\subsection{Event definition and selection}

The observational data are searched for signatures of small-scale dynamic events. For that purpose, the brightness temperature 


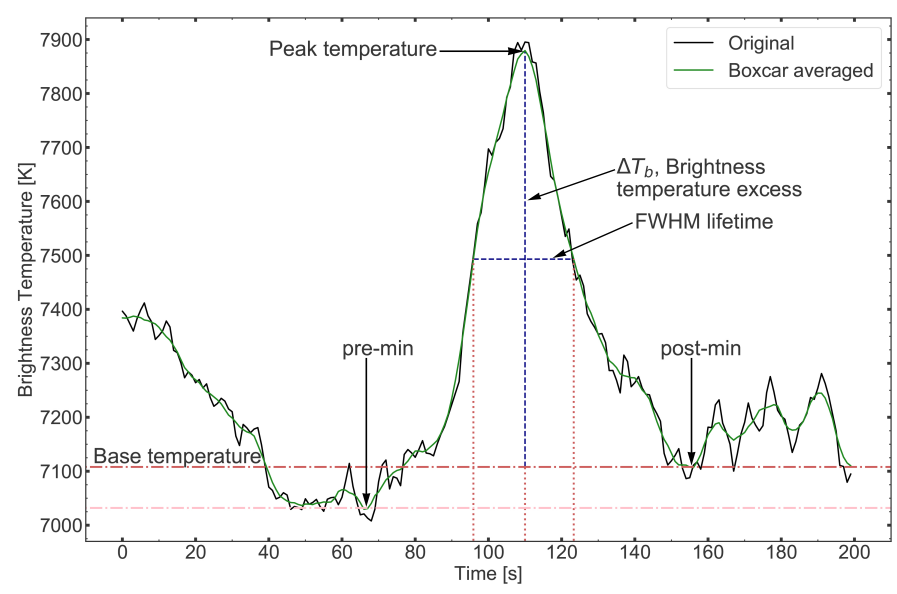

Fig. 1. Definition and selection criteria for a dynamic event. Temporal evolution of the brightness temperature at a selected pixel exhibits a peak enclosed by two minima (pre-min. and post-min.). The green graph shows the box-car average of the original data (black graph). The blue dashed lines mark the peak amplitude and FWHM lifetime. The horizontal red dashed-dotted line marks the temperature of the local minima with highest temperature; in this case the post-peak minima, which is the base brightness temperature considered for the specific event. The pink dashed-dotted line shows the temperature of the other minima; in this case the pre-peak minima. The red dotted lines mark the times where the temperature is at FWHM and peak.

The observational data are time evolution $\left(T_{\mathrm{b}}(t)\right)$ of each pixel is studied. To remove high-frequency noise, the data are boxcar averaged in time with a size of seven time steps $(14 \mathrm{~s})$. The resulting smoothed data are then searched for peaks in brightness temperature at every pixel, comprising an increase followed by a decrease in brightness temperature (cf. Fig. 1), with an amplitude of at least $400 \mathrm{~K}$. The amplitude of the peak in brightness temperature excess is measured as the difference from the base temperature level as determined by the closest significant local minima of $T_{\mathrm{b}}(t)$ at a given spatial position. To remain conservative, the minimum with the smallest $T_{\mathrm{b}}$ difference from the peak is chosen. The lifetime of an event is then determined as the FWHM of the brightness temperature excess peak (see Fig. 1).

The pixels showing such a temporary rise in brightness temperature are grouped together using the $k$-means clustering method (Everitt 1972). Each resulting cluster represents an individual event. The $k$-means clustering method sorts multidimensional data into a predefined number of clusters and then assigns the pixels to clusters depending on the euclidean distance in spatial and temporal coordinates with respect to the mean value for each cluster. The suitable number of clusters is determined by a combination of the following two methods:

(i) Start with a small number of clusters and perform the $k$-means clustering, calculate the entropy (i.e., the mean of the spread within the clusters), and then iteratively repeat the process with an increasing number of clusters. At last, pinpoint the number of clusters where the entropy does not drop significantly compared to previous steps with a smaller number of clusters. This method is commonly referred to as the "elbow method".

(ii) Compare each data point to its own cluster and to the closest neighboring cluster to see which cluster it is most closely related to. This method is commonly referred to as the "silhouette method".

In the central parts of the FOV, the events are more easily separated than when going towards the outer parts of the FOV. We conclude that this effect is caused by the radial increase of
$T_{\mathrm{b}}$ uncertainties resulting from the image reconstruction process of the interferometric measurements. The uncertainties becomes very pronounced in the outer parts of the FOV. Because of the lower reliability of the data in these parts, the $k$-means clustering is only applied to the innermost parts within a radius of $15^{\prime \prime}$.

In that inner region, all events are taken into account that (i) show clear peaks with a brightness temperature excess of more than $400 \mathrm{~K}$ and (ii) are fully represented in a single time scan. Thus, the length of the time scans $(10 \mathrm{~min})$ restricts the amount of events detected. Since the imaging procedures of solar ALMA data are still in an early stage, the criteria are defined more conservatively for this initial study in order to minimize the number of false detections.

\section{Results}

\subsection{Events with peaks in the temporal evolution of the brightness temperature}

A total number of 552 events are found within the inner parts of the FOV with a radius of $15^{\prime \prime}$ (see Sect. 2.2 for the selection criteria). The individual events are well distributed over almost the entire selected part of the FOV and all four time scans as can be seen from the spatial and temporal coordinates of the individual events in Fig. 2.

There are some parts, dominated by the top part as well as smaller segments in the bottom of the inner region, that do not show any events with brightness temperature variations of more than $400 \mathrm{~K}$ (Fig. 2d). This is because of the presence of strong magnetic fields and is studied further in Sect. 3.2.

The events have spatial sizes up to about 9" which corresponds to $\sim 4.3-6.4$ times the clean beam. The spatial size of an event is indicated by the maximum euclidean distance between the pixels within the event. However, the majority of events are smaller than $\sim 2.5^{\prime \prime}$, which can be seen in Fig. 3 where the distribution of spatial sizes is presented. From the peak value at $2.5^{\prime \prime}$, there is a steady decrease in the number of events towards larger sizes. Events smaller than the clean beam axes cannot be spatially resolved, resulting in a decrease in the occurrence rate towards smaller sizes. Pronounced events might be detected despite being not spatially resolved but a larger number of events with smaller sizes is expected.

For a specific event, each pixel has a well-defined time for the maximum peak brightness temperature which is plotted in panels $\mathrm{b}$ and $\mathrm{c}$ of Fig. 2. The lifetime and magnitude of the peak brightness temperature $\left(\Delta T_{\mathrm{b}}\right)$ vary between the pixels within a single event. These variations are caused by a combination of the inherent properties of the evolution of different parts of the events and the limited spatial resolution, which effectively smooths out the signatures. A more detailed discussion of these issues is provided in Sect. 4. For this study, the overall lifetime of the event is determined as the FWHM of the peak within the event with the largest $T_{\mathrm{b}}$ excess (cf. Fig. 1). The resulting values of lifetime and excess in $T_{\mathrm{b}}$ vary significantly among the individual events. The lifetimes range between $\sim 43$ and $360 \mathrm{~s}$ and the brightness temperature excess $\Delta T_{\mathrm{b}}$ between the cut-off limit of $400 \mathrm{~K}$ up to $\sim 1200 \mathrm{~K}$. A statistical analysis of these results is presented in Sect. 3.3.

\subsection{Magnetic field strength and network/internetwork mask of the entire FOV}

The observed quiet Sun region is composed of magnetic network (NW) and interwork (IN) regions (Wedemeyer et al. 2020). 


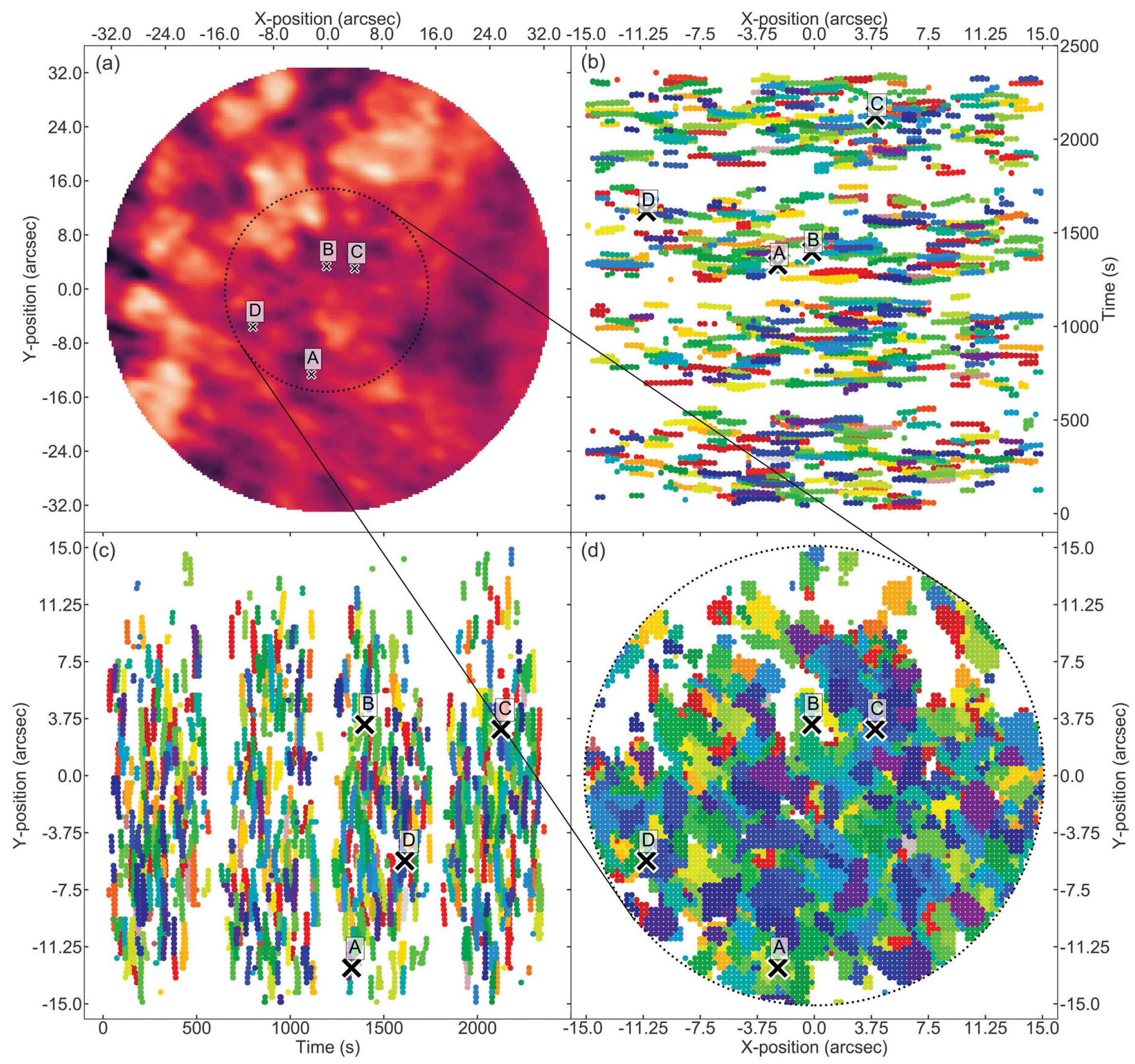

Fig. 2. Spatial and temporal position of all 552 detected temperature peak events within 15 arcseconds from the center of the FOV and with a magnitude of at least $400 \mathrm{~K}$. (a) Brightness temperature map of the first frame of the dataset $(2 \mathrm{~s}$ integration). The black dotted circle marks a radial distance of $15^{\prime \prime}$ from the center of the FOV. $(b)$ The $x$-axis position and $(c)$ the $y$-axis position of all brightness temperature peaks plotted against time, respectively. The events are all colored individually, showing the same color for the same event in all panels. $(d)$ The $x$-axis and $y$-axis positions of all temperature peaks. The locations of a few selected events (A, B, C and D) that are studied in detail in Sect. 3.4 are marked with crosses in all panels.

The magnetic field strength of the entire FOV, averaged in time over the ALMA observation period, is shown in the SDO/HMI magnetogram in Fig. 4a and ranges from -160 to $230 \mathrm{G}$. The strongest magnetic fields are present in the top parts of the FOV as well in the lower left corner. In the top right, there are two nearby locations with concentrations of opposite polarity. Between these locations, there are magnetic loop structures observable in many of the SDO-AIA channels. This group of compact loops is also seen in the ALMA Band 3 maps (Wedemeyer et al. 2020). The NW/IN mask by Wedemeyer et al. (2020) is overplotted in Fig. 4. This mask was composed based on time-averaged observational maps from SDO-AIA in 160 and $170 \mathrm{~nm}$ and saturated SDO/HMI magnetograms in combination with the ALMA maps. The locations of the stronger magnetic fields and their polarities are roughly stationary during the time period of the ALMA observations and therefore an average over the whole observational period is used when defining the different areas.

Using a NW/IN mask is a commonly used method for distinguishing between different types of regions, but as such represents a compromise across the considered observation period and different imprints of the network in the considered atmospheric 
H. Eklund et al.: The Sun at millimeter wavelengths. II.

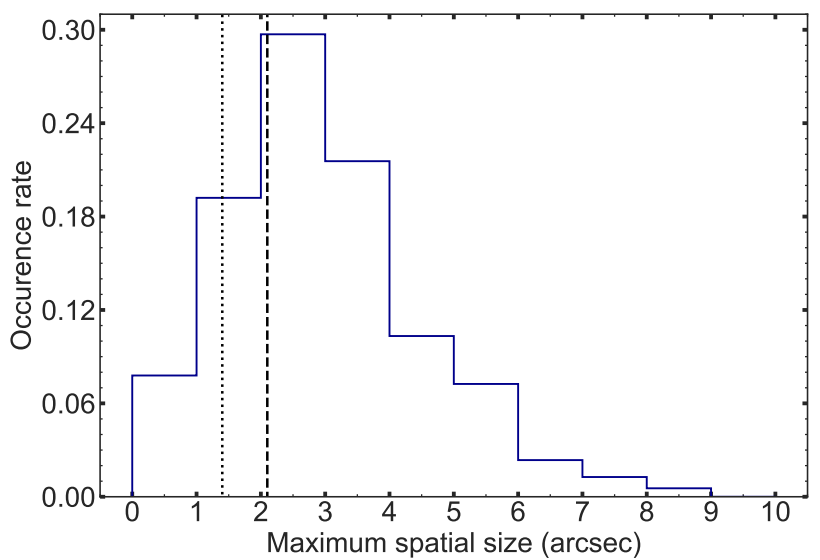

Fig. 3. Spatial size distribution of the 552 detected events, represented in bins with width of $1.0^{\prime \prime}$. The vertical dotted and dashed lines mark the sizes of the clean beam minor and major axis, respectively.

layers. Consequently, such a mask does not always adequately represent the conditions at the exact time and location of a specific dynamical event, such as those discussed here. The mask is shown nonetheless in order to facilitate comparisons with other studies using NW/IN masks (e.g. Loukitcheva et al. 2009) and with our previous paper (Wedemeyer et al. 2020).

In Fig. 4b, all pixels that show a peak with brightness temperature excess with a minimum amplitude of $400 \mathrm{~K}$ and a lifetime shorter than $200 \mathrm{~s}$ in the entire FOV and in all of the four time scans are marked. The contours of the time-averaged magnetic field strength and the NW/IN mask are overplotted for direct comparison. The occurrence of $T_{\mathrm{b}}$ peak signatures is much lower in the upper and left parts of the FOV, where the magnetic field strength is stronger on average, as well in a few patches at a radial distance of $\sim 15^{\prime \prime}$ towards the bottom. It should be emphasised that the magnetic field structure varies significantly over time on small spatial scales with nonaveraged line-of-sight (LOS) magnetic field strength spanning between [-260, 300] G. As a result, some $T_{\mathrm{b}}$ peaks appear to occur at locations with stronger time-averaged LOS magnetic field strength (Fig. 4b), while the actual magnetic field strength at the exact time of (and before) the occurrence of the peak is typically much lower. For this reason, a histogram for the LOS magnetic field strength at the exact location and the time of each $T_{\mathrm{b}}$ peak is shown in Fig. $4 \mathrm{c}$ in comparison to the distribution of all magnetic field strength values in the data set. The ratio of the two distributions seems to remain more or less constant for LOS magnetic field strengths below $\sim 180 \mathrm{G}$ but decreases with increasing magnetic field strengths beyond that point. This finding implies that the occurrence of brightness temperature peaks is reduced at locations with a LOS magnetic field strength of $\gtrsim 180 \mathrm{G}$ but the number of detections on which this conclusion is based is rather low. Furthermore, while the degree to which one can relate the SDO/HMI magnetograms measured at photospheric levels to the ALMA maps originating at larger heights is uncertain, it is valuable to point out that there are no $T_{\mathrm{b}}$ peaks at locations with absolute LOS magnetic field strengths greater than $\sim 250 \mathrm{G}$, which suggests that the presence of magnetic fields impacts the occurrence of the analyzed dynamic small-scale events.

\subsection{Statistical study of detected events}

For each of the 552 events (described in Sect. 3.1) the maximum $T_{\mathrm{b}}$ excess, lifetime, and base brightness temperature (cf. Fig. 1)
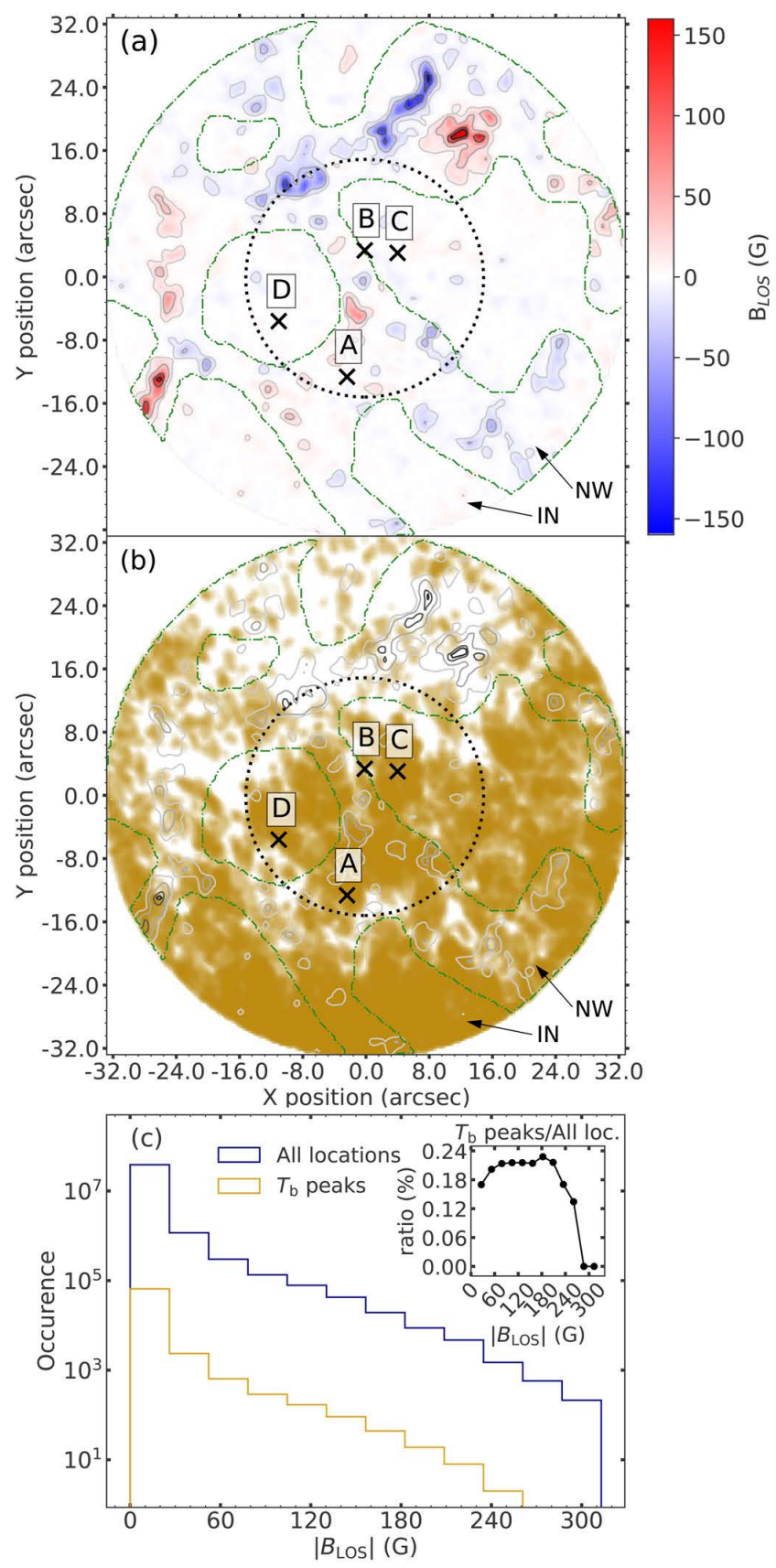

Fig. 4. Spatial comparison between brightness temperature dynamics in ALMA and SDO/HMI magnetogram. (a) The time-averaged SDO/HMI magnetogram for the whole ALMA FOV is presented as blue, white, and red shades with the value range saturated at $[-160,160] \mathrm{G}$. The absolute LOS magnetic field strength is outlined with gray contour lines with the levels $10,30,80$, and $120 \mathrm{G}$. The green dotted-dashed lines mark the mask defining the borders between network and internetwork regions. The dotted ring marks the radial distance of $15^{\prime \prime}$ from the center of the FOV. The spatial positions of the selected events A-D (Sect. 3.4 and Appendix B) are marked for reference. (b) The spatial position of all instances in the entire FOV with a $T_{\mathrm{b}}$ excess of more than $400 \mathrm{~K}$ and a lifetime shorter than $200 \mathrm{~s}$, at any time in the data, are marked with orange-brown dots. The white spaces are therefore locations without any events and a single pixel can show several $T_{\mathrm{b}}$ instances. The gray contour outlines the SDO/HMI magnetogram, whereas the green dotted-dashed contours, the dotted ring, and the crosses A-D mark the same as in panel $a$. (c) Histograms of the absolute LOS magnetic field strength at the locations and times of the $T_{\mathrm{b}}$ peaks marked in panel $b$ (orange-brown) and for the entire HMI data set (blue). The inset plot shows the ratio of the two histograms. 

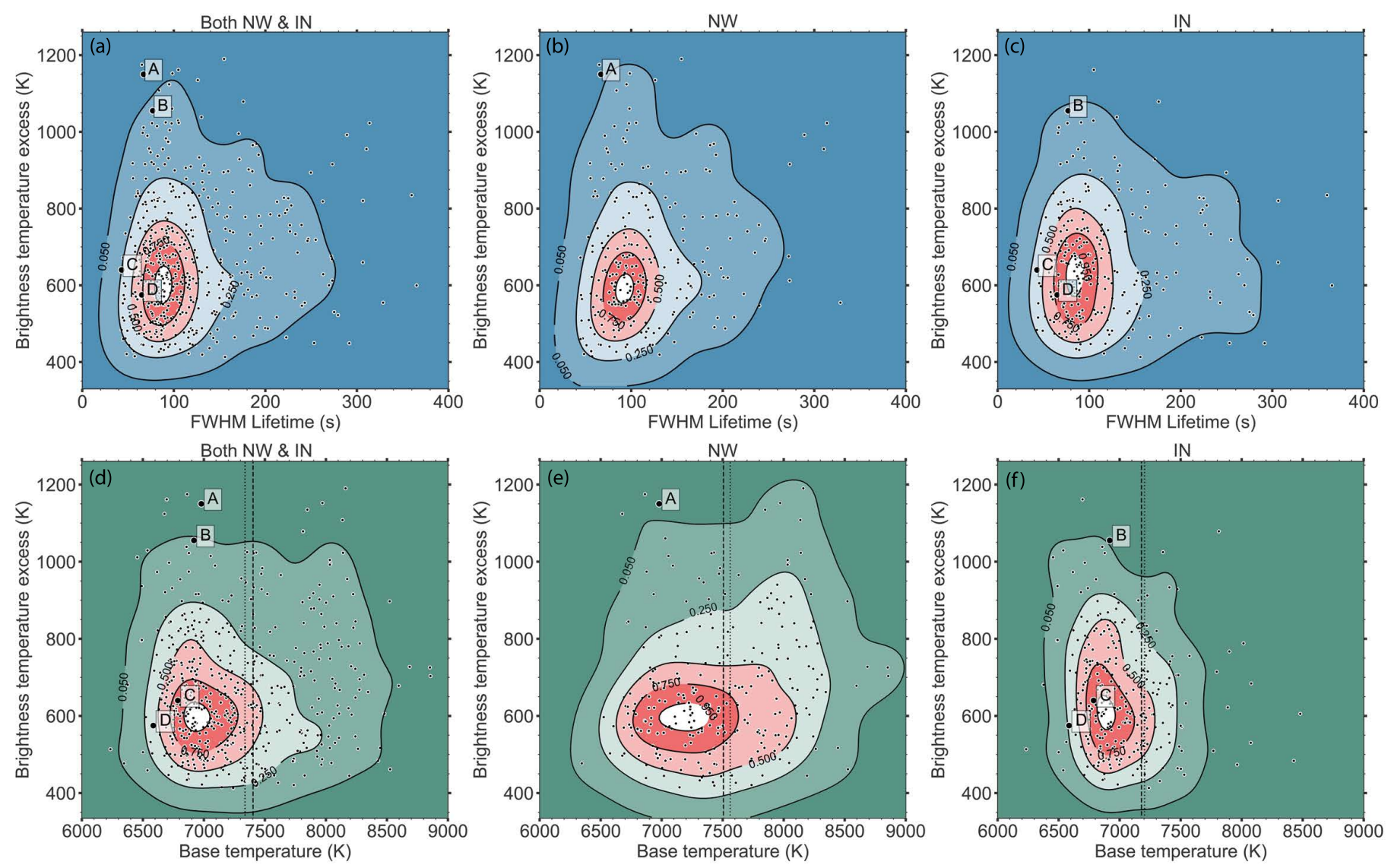

Fig. 5. Statistical study of all the events, with comparison between the regions defined by the NW/IN mask. The first column (panels $a$ and $d$ ) shows all the events, the second column (panels $b$ and $e$ ) shows the events within the NW mask, and the third column (panels $c$ and $f$ ) shows the events within the INmask. For each event, the first row shows the brightness temperature excess plotted against the lifetime and the second row shows the brightness temperature excess plotted against the base brightness temperature. The distributions are represented as density maps and the contour lines mark the levels of $0.05,0.25,0.5,0.75$, and 0.95 . The dotted and dashed lines in panels $d$, $e$, and $f$ show the median and mean value, respectively, of the brightness temperature of all pixels within 15" during the entire dataset. The selected events A-D (Sect. 3.4) are marked in all panels for reference.

are given in Fig. 5. As mentioned in Sect. 2.2, the $T_{\mathrm{b}}$ values in the outskirts of the FOV are less certain and events detected there are therefore not considered further for the detailed analysis of event properties. In the panels of the first column, all the events are plotted while the events are split up according to the NW/IN mask in the second (NW) and third (IN) columns. In all panels, the selected examples A-D are marked, which are described in detail in Sect. 3.4. In the first row, the lifetime of the events is plotted against their maximum brightness temperature excess $\left(\Delta T_{\mathrm{b}}\right)$. While the distribution in both lifetime and $\Delta T_{\mathrm{b}}$ appears to be continuous for all the events (Fig. 5a), there are a couple of noteworthy trends: ( $i$ ) The density distribution of the scatter plot indicates the most frequent events have a typical brightness temperature excess of $\Delta T_{\mathrm{b}} \sim 600 \mathrm{~K}$ and a lifetime of $\sim 90 \mathrm{~s}$, with values in the ranges of $\sim 450-750 \mathrm{~K}$ and $\sim 55-125 \mathrm{~s}$, respectively, for $50 \%$ of the distribution. (ii) There are no events with a lifetime below $43 \mathrm{~s}$ that also have a brightness temperature excess above $400 \mathrm{~K}$. This finding is discussed in connection with the effects of limited angular resolution in Sect. 4.2. The events that fall within the NW and IN areas of the mask shown in Figs. 5b and $5 \mathrm{c}$, respectively. The distributions of lifetime and brightness temperature excess for the NW and the IN areas are qualitatively similar but differ in a few aspects. Most of the relatively few events with larger $T_{\mathrm{b}}$ excess (above $\sim 1050 \mathrm{~K}$ ) are detected in the NW regions. However, this does not imply that stronger mag- netic field gives rise to larger $T_{\mathrm{b}}$ excess, as we see in Fig. 6. Similarly, the events with low $T_{\mathrm{b}}$ excess and long lifetime (up to $\sim 300 \mathrm{~s}$ ) tend to lie within the IN regions but this affects only a small number of events.

The base temperature (cf. Fig. 1) could potentially affect the $T_{\mathrm{b}}$ excess amplitude of an event. The brightness temperature excess is plotted against the base brightness temperature for all events in Fig. 5d. There is a continuous distribution of events with base brightness temperatures in the range of $\sim 6400-8300 \mathrm{~K}$ for all brightness temperature excess values $\Delta T_{\mathrm{b}}$ between $\sim 400$ and $1000 \mathrm{~K}$. The density plot in Fig. $5 \mathrm{~d}$ indicates that most events exhibit a base brightness temperature of around $6600-7500 \mathrm{~K}$. The mean and median brightness temperatures of all pixels within the same area as the considered events (15" radius and NW/IN respectively) for the entire time of the dataset are marked as references in Figs. 5d-f. The mean and median temperatures of the whole area are $\left\langle T_{\mathrm{b}}\right\rangle_{x, y, t}=7402 \mathrm{~K}$ and $7336 \mathrm{~K}$, respectively, which corresponds to the upper segment of the base temperatures shown for the events. This is naturally a cause of the base temperatures being defined at local minima of the $T_{\mathrm{b}}$ evolution. The separation of the events in NW and IN areas shows that there are more events with higher base temperature in the NW areas than in the IN areas (Figs. 5e-f. In accordance with higher (average) temperatures in the NW regions, the majority of events in the NW areas exhibit base 

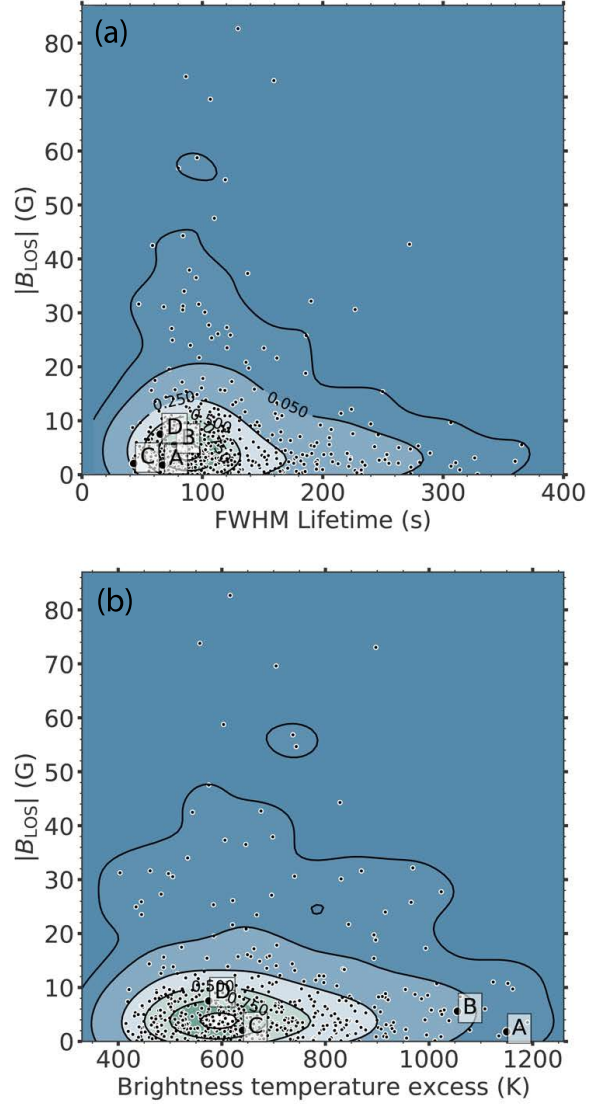

Fig. 6. Statistical study of all the events within $15^{\prime \prime}$ radius from the center FOV. (a) The absolute LOS magnetic field strength plotted against the lifetime for each event. (b) The absolute LOS magnetic field strength plotted against the $T_{\mathrm{b}}$ excess. The scatter plots are represented as colored density maps with the contour lines marking the levels of 0.01 , $0.05,0.25,0.5,0.75$, and 0.95 of the total distribution. The selected events A-D (Sect. 3.4) are marked in all panels for reference.

temperatures of up to $\sim 8100 \mathrm{~K}$, whereas there only a few events in the IN areas with such high values. The lower limit is however almost the same for the two masked areas, which comes naturally as the NW mask contains very quiet patches as well at some locations and times. For the NW regions alone, the mean and median values are $7561 \mathrm{~K}$ and $7506 \mathrm{~K}$, and for IN alone the values are smaller, $7204 \mathrm{~K}$ and $7179 \mathrm{~K}$, respectively. The mean of the brightness temperature is slightly higher than the median and is most pronounced in the NW areas, but is also evident in the IN areas, which suggests that there are only a few features but that these are very bright. Even though there is a significant shift towards larger base brightness temperature for the NW regions, the distribution of magnitude of the $T_{\mathrm{b}}$ excess is similar and there is only a mean brightness temperature difference of about $360 \mathrm{~K}$ between NW and IN areas. These results imply that the physical conditions behind the occurrence of the registered dynamical $T_{\mathrm{b}}$ excess events are not very dependent on base temperatures in the analyzed quiet Sun region.

As expected from the studies of other chromospheric diagnostics (see, e.g., de Wijn et al. 2009; Loukitcheva et al. 2009, and references therein), the (base) brightness temperature in ALMA Band 3 correlates with the (time-averaged) magnetic field strength in the photosphere (see Wedemeyer et al. 2020, for the analyzed data set). In Fig. 6, for each of the 552 events, the relations between the magnitude of the maximum brightness temperature excess $\Delta T_{\mathrm{b}}$, the lifetime, and the SDO/HMI

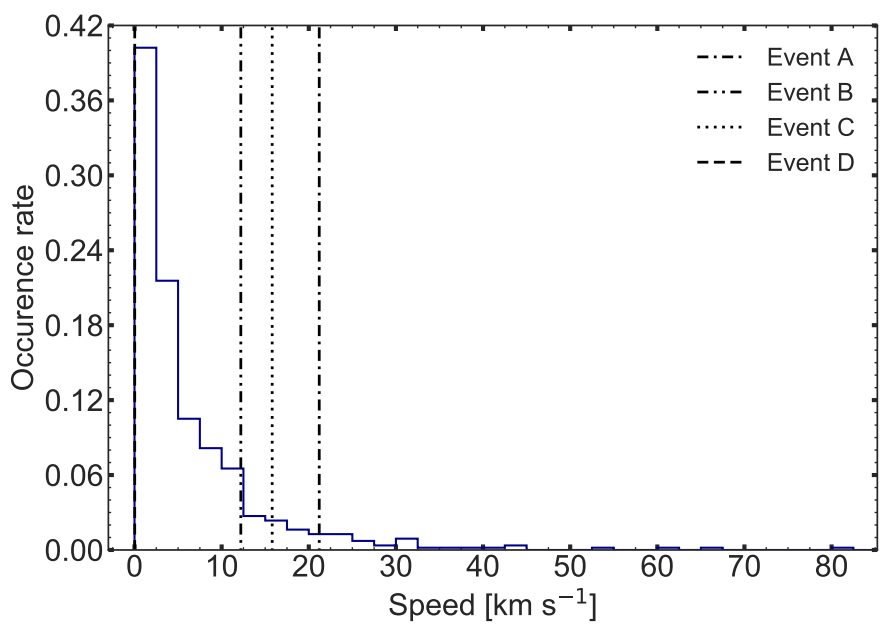

Fig. 7. Distribution of the speed of apparent motion of the 552 events. The speeds of events A-D (Sect. 3.4) are marked for reference.

LOS magnetic field strength are shown. The absolute LOS magnetic field strength is given at the spatial location and the time of the maximum excess $T_{\mathrm{b}}$ peak for each event. The absolute magnetic field strength at the locations of the detected events ranges up to $\sim 80 \mathrm{G}$. The majority of the events occur at locations with very low magnetic field strength below $10 \mathrm{G}$ and as many as $95 \%$ of the events are found at locations with an absolute magnitude of $\leq 20 \mathrm{G}$. There are some events with higher absolute magnetic field strength which are primarily occurring in the NW areas. However, even in the NW areas, the locations of the majority of the events show a low magnetic field strength similar to the events in the IN areas. This is because of the smallscale structure of the magnetic field unaccounted for by the timeaveraged NW/IN mask. The NW regions therefore still contain many areas with low average magnetic field strength, as can be seen in Fig. 4a, where there are many white patches also in the NW area. The events associated with larger absolute LOS magnetic field strength have shorter lifetimes, centered around $100 \mathrm{~s}$, similar to the majority of events. The events with longer lifetimes tend to occur at locations with a weak magnetic field. A similar but less clear trend can be seen in the brightness temperature excess with a larger spread of values for the events with larger magnetic field strength, similar to the distribution of the events with weak magnetic fields. These results may suggest that a strong LOS magnetic field disturbs the generation of events with long lifetime or higher amplitude $\left(\Delta T_{\mathrm{b}}\right)$. Even though the statistical sample is rather large with 552 events, a larger number of events with higher magnetic field strength is required in order to draw firm conclusions.

Apparent motion. In addition, the apparent motion is estimated for each event. This is done by looking at the change of position of the brightest point within the spatial extension of the event between the points in time at FWHM of the peak with largest $T_{\mathrm{b}}$. The individual velocity components in $x$ - and $y$-axis are found to be normally distributed around zero, which insinuates that there is no preferred direction of the apparent motion of the events. The probability density function of the total absolute apparent velocity is given in Fig. 7. Approximately $80 \%$ of the events show low speeds below $10 \mathrm{~km} \mathrm{~s}^{-1}$ and the rest show speeds between 10 and $30 \mathrm{~km} \mathrm{~s}^{-1}$, except for a handful of events with indications of high speeds of several tens of kilometres per second. 
Repetitiveness of events. Some of the events occur at similar locations but with a delay in time, which can be inferred from comparing and tracing individual events between the panels in Fig. 2. An estimation of the repetitiveness of events is made by taking the spatial and temporal locations of the points with largest magnitude of $T_{\mathrm{b}}$ excess in each event and calculating how many of these are within a certain spatial distance from each other. Approximately $22 \%$ of the events occur within a spatial distance of $1^{\prime \prime}$ and within the same time scan (of $\sim 10 \mathrm{~min}$; Sect. 2.1) as another event. For these events occurring at closeby locations, the delay between the occurrence of the brightness temperature peaks of two events is typically $\Delta t_{\text {peaks }}=200 \mathrm{~s}$, although a continuous distribution of delays of up to approximately $\Delta t_{\text {peaks }}=320 \mathrm{~s}$ and in a few cases even up to $\Delta t_{\text {peaks }}=$ $430 \mathrm{~s}$ is found. It should be noted that the statistical significance of the derived delays is limited by the duration of the observational time scans and the spatial threshold (here set to $1^{\prime \prime}$ ) in relation to the angular resolution. The complexity of the 3D structure of the solar chromosphere on small spatial scales in combination with the limited angular resolution can significantly affect the observable signatures of dynamic events at mm wavelengths (see the discussion in Sects. 4.2 and 4.3). Consequently, propagating shock waves that are excited at photospheric heights can appear very different even if they were excited at the same location. Indications of the repetitiveness of events can be seen in the time evolution graphs of the selected examples in Figs. 8-9 and B.1-B.2.

\subsection{In-depth study of selected events in the observational data}

A couple examples of dynamic events, representative for different segments of the parameter space (see Figs. 5 and 6), are illustrated in Figs. 8 and 9. Event A exhibits more pronounced brightness temperature amplitude and event $\mathrm{C}$ shows a weaker $T_{\mathrm{b}}$ excess, representative of the most frequently detected events (as seen in Fig. 5a). An additional two examples (events B and D), showing similar signatures yet with different details, are presented in Appendix B. All four events are marked in Figs. 2-6 for reference.

Event A, which is located at $(x, y)=\left(-2.4^{\prime \prime},-12.6^{\prime \prime}\right)$, is shown in Fig. 8. The lower right panel h shows the time evolution of the brightness temperature at a fixed location with a rise from $T_{\mathrm{b}}=6980 \mathrm{~K}$ to $8130 \mathrm{~K}$ over about $53 \mathrm{~s}$. After reaching the peak, the brightness temperature decreases again to $6940 \mathrm{~K}$ over the course of $67 \mathrm{~s}$, to only slightly below the initial $T_{\mathrm{b}}$ value. Event $\mathrm{A}$ therefore has a temperature excess of $\Delta T_{\mathrm{b}}=1150 \mathrm{~K}$ and a lifetime of $67 \mathrm{~s}$. The area connected to the event, for example the area that shows a brightness temperature of at least half the maximum of the $\Delta T_{\mathrm{b}}$ peak, has a round shape with a maximum diameter of roughly 4 arcsec. The upper row with five panels shows a time series of close-ups of the surroundings of the event. The event develops rapidly at one location in the FOV and expands in all directions and then decreases in brightness whilst remaining relatively (horizontally) stationary apart from a rightward motion component. Even though the limited angular resolution makes it challenging, the apparent movement through time of the brightest point, of approximately $22 \mathrm{~km} \mathrm{~s}^{-1}$, can be seen in the space-time diagrams in the lower left panels (Figs. 8fg). As indicated in Fig. 4, event A is located in the NW region, but still shows a weak absolute magnetic field strength of only $\sim 18 \mathrm{G}$. In the $T_{\mathrm{b}}$ time evolution (Fig. $8 \mathrm{~h}$ ), two weaker succeeding peaks can be seen approximately $4.5 \mathrm{~min}$ and $7 \mathrm{~min}$ after the strong peak of event A. Even though these specific peaks might not fulfill the $\Delta T_{\mathrm{b}}>400 \mathrm{~K}$ requirement used in the selection of the events (Sect. 2.2), the time delay is in line with the numbers presented in Sect. 3.3 for the repetitiveness of events. Another strong event B showing many similarities to A is illustrated in Fig. B.1 for reference.

Event C (Fig. 9) occurs at the location $(x, y)=\left(4.0^{\prime \prime}, 3.0^{\prime \prime}\right)$ close to event $\mathrm{B}$, but more than 6 minutes later in time. The brightness temperature increases from $6790 \mathrm{~K}$ to $7430 \mathrm{~K}$, resulting in $\Delta T_{\mathrm{b}}=640 \mathrm{~K}$ and a lifetime of $43 \mathrm{~s}$. After reaching the $T_{\mathrm{b}}$ peak, there is a significant drop to about $6200 \mathrm{~K}$. As can be seen in the space-time diagrams in the lower left panels, the temperature in the area drops to about $6000 \mathrm{~K}$. We conclude that this more pronounced contrast is likely caused by the formation of a slightly larger-than-average post-shock region. In the ALMA data presented here, such regions are typically seen as round shapes with diameters on the order of up to a few arcsec. However, most of these regions have typical diameters of $1-2^{\prime \prime}$, which is at or below the resolution limit in the ALMA Band 3 data used here. The post-shock region occurring in connection to event $\mathrm{C}$ is slightly larger and therefore better resolved, revealing a larger $T_{\mathrm{b}}$ contrast. See Sects. 4.2 and 4.3 (and in addition, e.g., Wedemeyer-Böhm et al. 2007; Loukitcheva et al. 2015) for a discussion on the impact of low spatial resolution on observations of propagating shock wave patterns. The bright feature appears stable in the horizontal plane with just a subtle movement towards higher values on the $x$-axis (Fig. 9). The speed of the apparent motion is $\sim 15 \mathrm{~km} \mathrm{~s}^{-1}$ at FWHM. Event D, which is similar to event $\mathrm{C}$ but supposedly even more on the limit of being resolved, is presented in Fig. B.2.

As we discuss in Sect. 4.3, the angular resolution achieved for the analyzed ALMA Band 3 observations is at the limit for resolving the chromospheric shock-wave-induced pattern. In areas with short distances between neighbouring shock waves, most of the resulting mesh-like pattern remains unresolved. There, the shock peaks do not stand out from the surroundings, but instead the observed dynamical structure is dominated by the darker (post-shock) regions. The latter example, event $C$, indeed shows less clearly isolated features (similar to event D in Fig. B.2). This could contribute to the apparent lower values of the most represented $\Delta T_{\mathrm{b}}$ amplitudes than what would be seen with higher angular resolution.

\section{Discussion}

\subsection{One-dimensional numerical simulations of propagating shock waves}

In order to check whether or not propagating shock waves could be producing the observed brightness temperature events, we study numerical simulations and calculate the radiative transfer in mm wavelengths. The hydrodynamic code RADYN (Carlsson \& Stein 1992, 1997, 2002, 1994) produces onedimensional dynamical model atmospheres where a moving piston in the lower boundary excites waves. RADYN solves the equations of conservation of energy, momentum, and mass, the radiative transfer equations in nonLTE, and the rate equations of atomic level populations. Loukitcheva et al. (2004, 2006) present $T_{\mathrm{b}}$ signatures at $\mathrm{mm}$ wavelengths resulting from RADYN runs for which the waves are excited by the driving piston with velocities set to match photospheric Doppler velocities obtained from observations in the Fe I line at $\lambda=396.68 \mathrm{~nm}$ (Carlsson \& Stein 2002). In the time series, with lengths of $\sim 1 \mathrm{~h}$, waves with varying strength and speed are excited, allowing some of them to interact. The resulting shock wave signatures at 
H. Eklund et al.: The Sun at millimeter wavelengths. II.

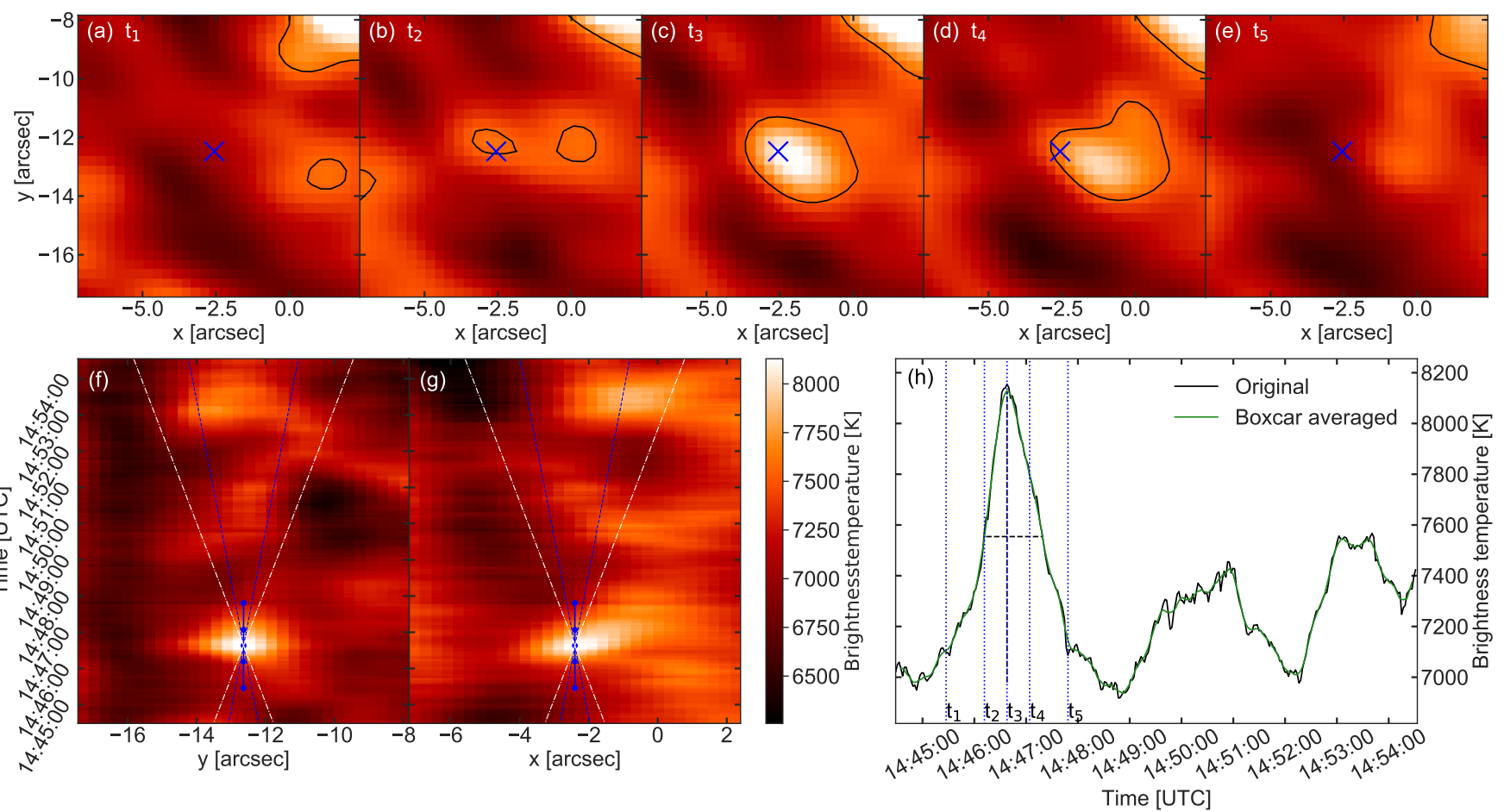

Fig. 8. Detailed study of event A. (a)-(e) The top panels show a close up of the surroundings at different time steps, $t_{1}$ to $t_{5}$ from left to right, through the shock wave event. The time of the $T_{\mathrm{b}}$ peak is marked by $t_{3}=14: 46: 38$. Here, $t_{1}$ and $t_{2}$ mark $70 \mathrm{~s}$ and $26 \mathrm{~s}$ prior the peak and $t_{4}$ and $t_{5}$ mark $26 \mathrm{~s}$ and $70 \mathrm{~s}$ after the peak. The center location is marked by a blue cross. The contour lines mark half of the maximum $\Delta T_{\mathrm{b}}$ peak. $(f)-(g)$ Space-time diagrams for a vertical and horizontal slit across the FOV at the center location, which is marked with blue dots for the time steps $t_{1}$ to $t_{5}$. Velocity slopes for 10 and $20 \mathrm{~km} \mathrm{~s}^{-1}$ are indicated by blue dotted and white dashed lines, respectively. The color code is the same in all panels. (h) The time evolution of the brightness temperature of the center location, where the time steps $t_{1}$ to $t_{5}$ are indicated by blue dotted vertical lines. Both the original data (black) and the averaged data (green) are shown. The horizontal and vertical black dashed lines mark the event lifetime and brightness temperature excess.
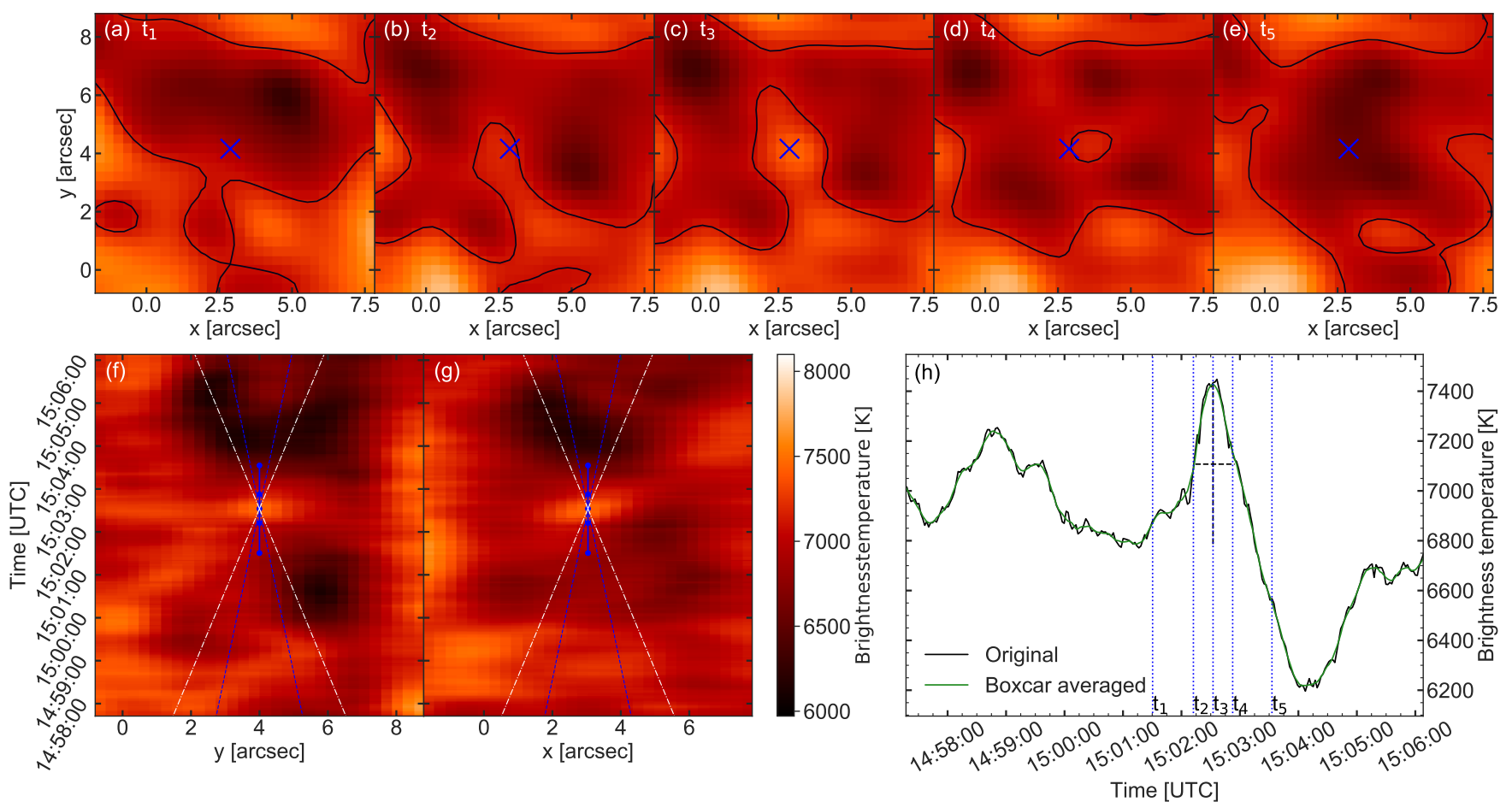

Fig. 9. Detailed study of event $\mathrm{C}$, as provided for event $\mathrm{A}$ in Fig. 8. The time of the $T_{\mathrm{b}}$ peak is marked by $t_{3}=15: 02: 32$. Here, $t_{1}, t_{2}, t_{4}$ and $t_{5}$ mark $-60,-20,20$, and $60 \mathrm{~s}$ from the peak, respectively. 
a wavelength of $3.4 \mathrm{~mm}$ (see Fig. 1c in Loukitcheva et al. 2006), which is close to the wavelengths currently offered for solar observing with ALMA in Band 3, show $\Delta T_{\mathrm{b}}$ amplitudes of between a few hundred $\mathrm{K}$ to $\sim 4000 \mathrm{~K}$ and lifetimes in the range of approximately 15-50 s. Some events are double-peaked, showing a long lifetime, which seems to be the result of interacting waves. Wave interference can therefore significantly contribute to extending the apparent lifetime of an event. In order to mitigate any input bias of the observational Doppler velocities, we perform a parameter exploration with a series of monochromatic waves covering large parameter ranges. This allows us to see under which circumstances shock waves can give rise to $T_{\mathrm{b}}$ signatures that are in line with the observed signatures described in Sect. 3.3. The RADYN code is used and the initial conditions are set by a VAL model atmosphere (Vernazza et al. 1981) with the addition of a transition region and corona (Carlsson \& Stein 2002). The lower boundary is set to $100 \mathrm{~km}$ below where the optical depth is unity at a wavelength of $500 \mathrm{~nm}$. The upper boundary is at a height of $10^{4} \mathrm{~km}$, where the temperature is set to $10^{6} \mathrm{~K}$. To resolve small-scale variations arising from the propagation of shock waves, an adaptive mesh is used (Dorfi \& Drury 1987). The duration of the simulations is $1500 \mathrm{~s}$ and thus long enough for the relaxation phase of the atmospheres to pass and for several shock waves to propagate through. The cadence of the output is $1 \mathrm{~s}$. The piston at the lower boundary excites sinusoidal acoustic waves where the initial amplitude and the periodicity are varied independently.

The amplitude is varied between 0.001 and 0.15 of the local sound speed, $v_{0}$, at the lower boundary, and the periodicity is varied between a few seconds up to several minutes. Distinct shock waves are excited with an initial amplitude of between $0.005 v_{0}$ and $0.05 v_{0}$ and wave periodicity between $P=90$ and $210 \mathrm{~s}$. The waves steepen into shock waves around $1 \mathrm{Mm}$ and propagate through the chromosphere with a speed of approximately $9.4 \mathrm{~km} \mathrm{~s}^{-1}$. The radiative transfer through the dynamical atmosphere models is calculated using the RH 1.5D code (Uitenbroek 2001; Pereira \& Uitenbroek 2015). The output continuum intensities $\left(I_{v}\right)$ are converted to brightness temperatures through relation (1).

For each time-step, the mean value of the brightness temperatures of all four sub-bands of ALMA band 3 (see Table 1) is calculated to match the spectral setup of the full band integrations in the ALMA observations, which makes a one-toone comparison possible. The temporal evolution of the simulated Band 3 brightness temperature is shown for the different simulation runs in Fig. 10. Each shock wave signature consists of a rapid increase in brightness temperature, reaching a peak, and followed by a post-shock drop in brightness temperature. The case of a static unperturbed atmosphere is given as a reference.

We note that, when going towards lower initial wave amplitudes, a preceding temperature peak is growing stronger and partly merges with main peak. The presence of a preceding peak is most prominent in the case of $A_{i}=0.005 v_{0}$, where the minimum brightness temperature is significantly enhanced in connection with the main peak for more than $60 \mathrm{~s}$, as well as throughout the entire cycle. Such double-peaked temporal profiles are also seen in the series with different wave modes (Loukitcheva et al. 2004, 2006), as a result of interference between waves. We also note that some of the observational examples, specifically event $\mathrm{C}$ (Fig. 9), exhibit a very subtle increase in $T_{\mathrm{b}}$ preceding the main peak, which might be consistent with the small preceding secondary peaks seen in the simulations for certain parameters.

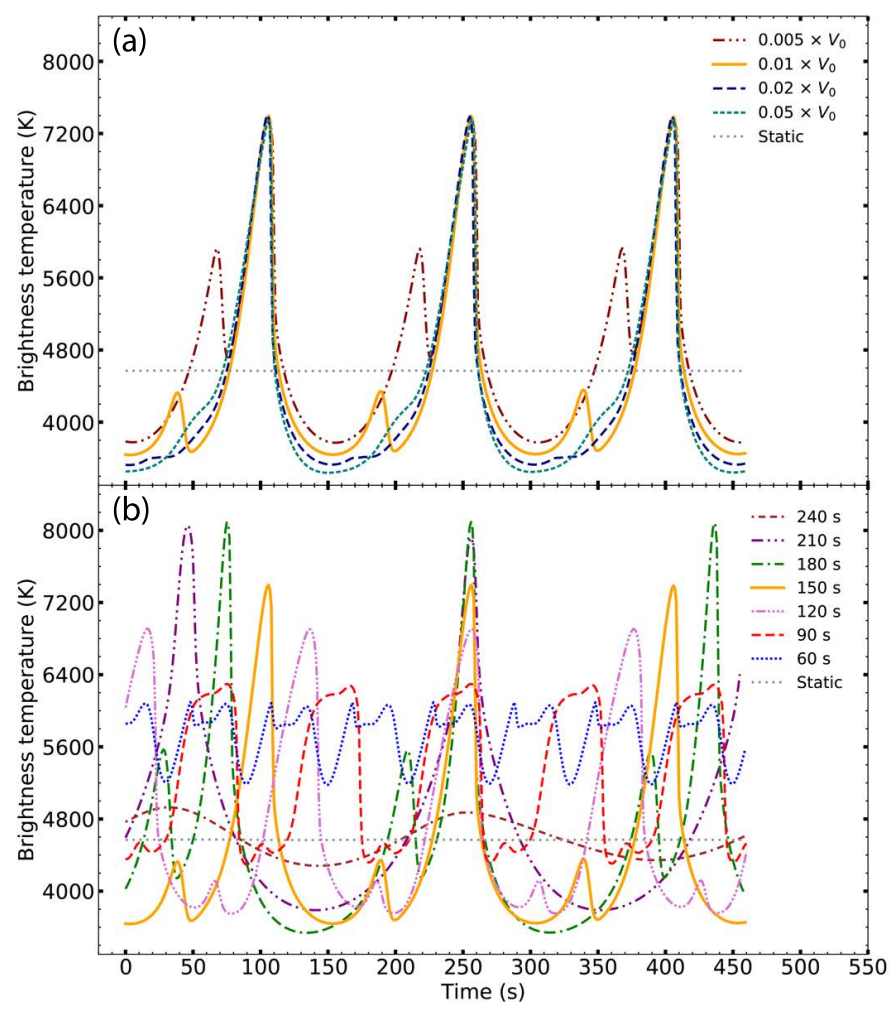

Fig. 10. Evolution of brightness temperature with propagating shock waves. (a) Variation of the initial wave amplitude $A_{i}$ between $0.5 \%$ and $5 \%$ times the local sound speed at the lower boundary $\left(v_{0}\right)$. The periodicity is kept at $150 \mathrm{~s}$. (b) Variation of the wave periodicity between 60 and $240 \mathrm{~s}$, where $A_{i}$ is kept at $0.01 v_{0}$. The orange graph in panels $a$ and $b$ represents the same run; in both panels, the case of a static atmosphere without any disturbances is plotted as reference with a gray dotted line. The runs are shifted in time to align the time of their peaks.

Figure 11 shows the brightness temperature excesses and the lifetimes of the shock wave signatures. The parameter exploration reveals that the variations of the initial amplitude only have a small impact on both the brightness temperature excess $\Delta T_{\mathrm{b}}$, which is found to be on the order of $3500-3900 \mathrm{~K}$ and on the lifetimes, with values of between 21 and $25 \mathrm{~s}$. In contrast, the wave periodicity has a large impact on the brightness temperature excess and signature lifetime (Fig. 11, panels a and c). In the case of long periodicity of $P=240 \mathrm{~s}$, the $T_{b}$ signature shows a low brightness temperature excess of only about $\sim 550 \mathrm{~K}$ with a corresponding long lifetime of $\sim 100 \mathrm{~s}$. This agrees with the results of Loukitcheva et al. (2006), where they show that at wavelengths of ALMA band 3, the intensity in the power spectrum at $240 \mathrm{~s}(4.2 \mathrm{mHz})$ is very low whilst the peak is found at a period of $175 \mathrm{~s}(5.75 \mathrm{mHz})$, which agrees well with Fig. 11a. A short periodicity of $P=60 \mathrm{~s}$ gives rise to $\Delta T_{\mathrm{b}}=900 \mathrm{~K}$ and a lifetime of $40 \mathrm{~s}$. However the bulk shift towards higher temperatures (Fig. 10b) indicates that the atmosphere is not allowed to relax between the passages of waves. For the cases between $P=90 \mathrm{~s}$ and $210 \mathrm{~s}$, with clear $T_{\mathrm{b}}$ shock wave signatures, values of the brightness temperature excess of between $\sim 2000 \mathrm{~K}$ and $4450 \mathrm{~K}$ are seen, together with lifetimes of between $16 \mathrm{~s}$ and $45 \mathrm{~s}$. This is consistent with the results from the series of waves derived from photospheric Doppler velocity measurements (Loukitcheva et al. 2006).

In conclusion, shock waves with reasonable wave parameters in agreement with observational constrained values give rise 


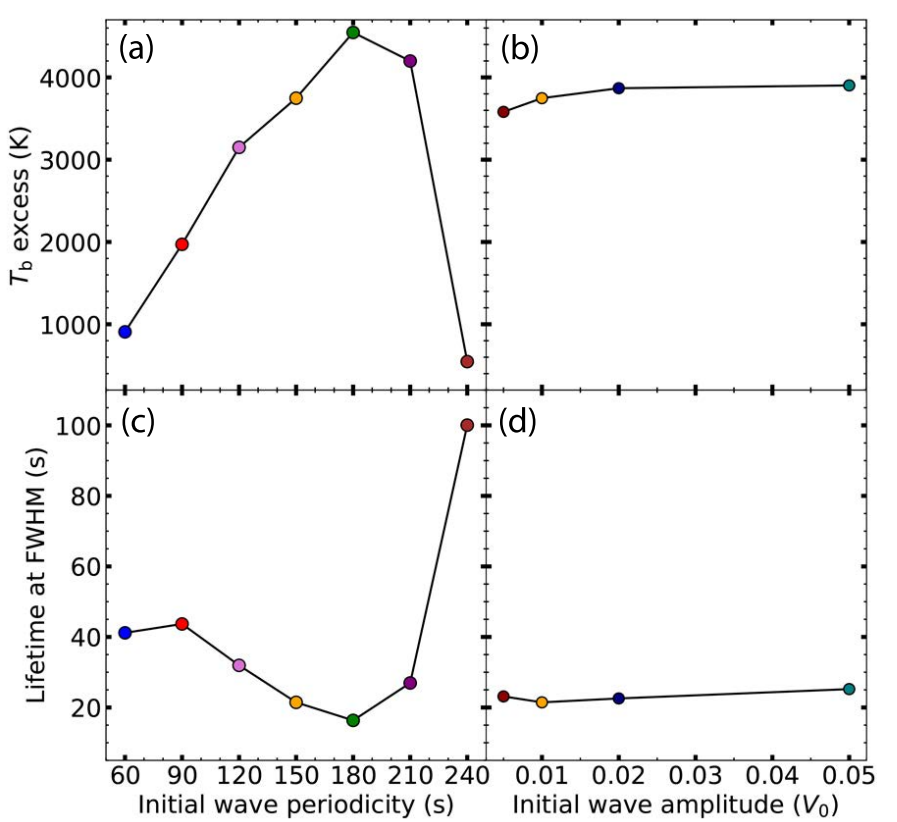

Fig. 11. Change of temperature and lifetime of shock wave signature with varying wave parameters. (a) Change of temperature as a function of initial wave frequency. ( $b$ ) Change of temperature as a function of initial wave amplitude. (c) Lifetime at FWHM of the brightness temperature excess peak as a function of initial wave frequency. (d) $T_{\mathrm{b}}$ peak lifetime as a function of initial wave amplitude. The color scheme is the same as in Fig. 10.

to signatures with brightness temperature excess $\Delta T_{\mathrm{b}}$ from a few hundred Kelvin up to approximately $4500 \mathrm{~K}$ (at wavelengths of ALMA band 3). $\Delta T_{\mathrm{b}}$ of $4500 \mathrm{~K}$ are about three to four times larger than what is seen in the observational ALMA data. In the following section we explore a possible explanation for this.

\subsection{Shock wave propagation in a 3D environment}

The one-dimensional simulations are only an idealized first approximation, whereas for shock wave signatures in the observational data the following two factors have to be considered: ( $i$ ) Many of the shock wave signatures are not fully resolved and there is spatial smearing with the surroundings that comes from sampling with the relatively large clean beam. Wöger et al. (2006) demonstrated that the dynamical timescale of the chromospheric small-scale internetwork structure does indeed increase when lowering the spatial resolution. (ii) The shock waves propagate through an inhomogeneous three-dimensional medium that is shaped by previous waves and magnetic fields. The shock waves can also interfere with each other and a fully isolated shock wave event as in the onedimensional simulations might be difficult to observe. Both constructive and destructive interference could change the observed brightness temperature.

Propagation of shock waves in a more realistic 3D environment is demonstrated here by means of numerical simulations with the Bifrost code (Gudiksen et al. 2011; Carlsson et al. 2016); see also Eklund et al. (2020) for a detailed analysis of an exemplary shock wave event. Here, we study the observational imprint of shock wave propagation in brightness temperature maps calculated with the Advanced Radiative Transfer (ART) code (de la Cruz Rodriguez et al., in prep.) at wavelengths corresponding to ALMA band 3 (cf. Table 1). The ART code assumes
LTE but takes into account relevant sources of continuum opacity in detail.

To estimate the magnitude of the degradation that comes with limited spatial resolution, the maps are degraded to the resolution of the ALMA band 3 observations by convolution with the clean beam (Sect. 2.1). An original $T_{\mathrm{b}}$ map for a selected time step is compared to the corresponding spatially degraded map in Figs. 12a-b). As expected, the fine structure on spatial scales smaller than the resolution limit is lost in the spatially degraded map. The location of an example of a propagating shock wave is marked in the respective mm maps. The evolution of the gas temperature surrounding the shock front is given in Fig. A.1 for reference. The time evolution of the brightness temperature at that location is shown in Fig. 12c for both the original map and the spatially degraded map. The propagating shock wave gives rise to a signature with a magnitude of $\Delta T_{\mathrm{b}}=3600 \mathrm{~K}$ in the map with original resolution. In the map with degraded spatial resolution, the shock wave shows a signature with a substantially reduced magnitude of $\Delta T_{\mathrm{b}}=640 \mathrm{~K}$. The limited spatial resolution of the ALMA band 3 observations therefore results in an observable signature of this shock wave example, which has $\sim 0.18$ times the original $\Delta T_{\mathrm{b}}$ magnitude. Applying this conversion factor to the temperature excess seen in the 1D simulations with values between $\sim 2000 \mathrm{~K}$ and $4450 \mathrm{~K}$ would result in reduced values of $\sim 360 \mathrm{~K}-800 \mathrm{~K}$. These values agree very well with what is seen in the observational data (Fig. 5), therefore implying that the observed signatures could be caused by propagating shock waves.

This is also in line with what Wedemeyer et al. (2020) demonstrated using the same Bifrost model. These latter authors find that applying the Band 3 synthetic beam reduces the brightness temperature standard deviation $T_{\mathrm{b}}^{\mathrm{rms}}$ from $1794 \mathrm{~K}$ to $1254 \mathrm{~K}$ for NW pixels and from $1304 \mathrm{~K}$ to $693 \mathrm{~K}$ for IN pixels, respectively, which is a factor of 0.70 for NW and 0.53 for IN. Applying these factors to the range of $900 \mathrm{~K}$ to $4600 \mathrm{~K}$ found with the 1D RADYN/RH simulations in Sect. 4.1 reduces the expected range of brightness temperature amplitudes in shock waves to $630 \mathrm{~K}-3220 \mathrm{~K}$ for NW pixels and to $480 \mathrm{~K}-2440 \mathrm{~K}$ for IN pixels. We note that interpolating the resolution-dependent $T_{\mathrm{b}}^{\mathrm{rms}}$ values by Loukitcheva et al. (2015) to the resolution of the Band 3 data (here $1.4^{\prime \prime}-2.1^{\prime \prime}$ ) also results in a reduction by a factor $\sim 0.7$ with respect to the original resolution. Wedemeyer-Böhm et al. (2007) also investigated the impact of the spatial resolution on their simulated brightness temperature maps showing a smallscale shock wave pattern, although for a shorter wavelength of $1 \mathrm{~mm}$. Using their Eq. (9) with a characteristic length scale of $D=1000 \mathrm{~km}$ and the angular resolution of the Band 3 data used here $\left(2.1^{\prime \prime}-1.4^{\prime \prime}\right)$ results in a reduction by a factor $0.22-$ 0.36 . The expected range of shock wave brightness temperature amplitudes based on the RADYN/RH results presented here would then be reduced to a lower limit of $200 \mathrm{~K}-330 \mathrm{~K}$ and an upper limit of $1000 \mathrm{~K}-1700 \mathrm{~K}$, which is much more in line with the range of values found in the observations in Sect. 3.3 (see Fig. 5).

The lifetime of the shock wave example is $70 \mathrm{~s}$ in its original resolution, and $61 \mathrm{~s}$ in the degraded resolution (Fig. 12c). The cooler pre-shock period around $t=1000 \mathrm{~s}$ is not apparent in the ALMA band 3 resolution which contributes to a shorter apparent lifetime. This example illustrates the fact that the lifetime of an event is heavily dependent on the surrounding dynamical structure and the extent (and orientation) of spatial smearing due to limited angular resolution. The resulting longer lifetimes seen in the $3 \mathrm{D}$ model and the observations as compared to the shorter lifetimes in the 1D simulations (Figs. 10 and 11) emphasises the 
shortcomings of the 1D approach in this particular aspect and the need for a systematic study based on 3D simulations.

The 1D RADYN simulations predict lifetimes for shock wave signatures in the range from $15 \mathrm{~s}$ to $50 \mathrm{~s}$ (see Sect. 4.1). The simulations also show that it is possible that hydrodynamical wave motions, although not steepening into shock waves, could show longer lifetimes on the order of $\sim 100$ s with a brightness temperature excess of at least $400 \mathrm{~K}$ (Fig. 10). The simulations indicate short-lived signatures well under one minute for shock waves. However, with a potential broadening of the temporal profiles, longer lifetimes are expected. The extent of this broadening is strictly dependent on the spatial smearing of the radiative maps, that is, the evolution of the surroundings of the signatures and the size of the clean beam.

While it is evident that the observable signatures in Band 3 brightness temperatures are highly dependent on resolution, it is also clear that a detailed comparison of simulated and observed signatures and a possible identification of shock waves as their source require a more direct approach using detailed 3D simulations (see Sect. 4.6).

\subsection{Detection of events with limited spatial resolution}

Even though many dynamical events with brightness temperature amplitudes of at least $400 \mathrm{~K}$ are detected, the distribution of the spatial sizes of the events (see Fig. 3) is most likely cut off at spatial scales corresponding to the size of the clean beam. It is plausible that the distribution can be extrapolated towards smaller sizes and that smaller events thus remain unresolved.

This assumption is supported by our study of the Bifrost simulation (Fig. 12), observations in other chromospheric diagnostics, for example in the $\mathrm{Ca}$ II $\mathrm{K}$ line at a spatial resolution of 0.7" (Wöger et al. 2006), and other numerical simulations (e.g., Wedemeyer et al. 2004) that clearly show small-scale features beyond the resolution of the ALMA Band 3 data analyzed here. Similar studies to that presented here, using measurements with higher spatial resolution, would provide further insight. A higher spatial resolution with the same wavelengths could be achieved by making measurements with a more extended antenna array configuration.

As mentioned in Sect. 1, the typical spatial scale of the meshlike pattern in the chromosphere originating from propagating shock waves is of the same order as the angular resolution of the ALMA data used here (Wedemeyer et al. 2004; Wöger et al. 2006; Wedemeyer-Böhm et al. 2007; Loukitcheva et al. 2015). Consequently, the limited spatial resolution of this dataset (1.4$2.1 \mathrm{arcsec}$ ) does not allow us to fully resolve the (sub)structure of the detected events, and potentially fails to detect small and weak events. Using the above-mentioned clustering technique, the 552 detected dynamical events in this dataset (within 15" radius) must therefore be considered to represent a lower limit only. Rather, only the strongest events and events with strong contrast with respect to their surroundings are detected. The effect of low angular resolution on the visibility of the chromospheric small-scale pattern is demonstrated by Wedemeyer-Böhm et al. (2007), Loukitcheva et al. (2015), and Wedemeyer et al. (2020).

The spatial resolution of interferometric data is often improved by the Earth-rotation aperture synthesis technique (Ryle 1975). Although this is an ingenious technique, it is inherently limited to a temporal span below that of the typical dynamical timescale of the target, as otherwise information is lost. In the case of the highly dynamical chromosphere, the dynamical timescale is on the order of seconds. To properly resolve dynamical events we therefore require a cadence of a couple of seconds at the most.

\subsection{Dependence on magnetic fields}

The results of this work show that the dynamical brightness temperature signatures are not evenly distributed in the FOV but that strong magnetic fields coincide with a lower occurrence of $T_{\mathrm{b}}$ signatures (see Fig. 4). Despite the difference in formation heights between the (chromospheric) $T_{\mathrm{b}}$ signatures in the ALMA band 3 data and the (photospheric) LOS magnetic field strength given by the SDO/HMI magnetograms, a lack of $T_{\mathrm{b}}$ signatures is seen, specifically at the footpoints of stronger magnetic field structures. It should be emphasized again that it is important to specifically refer to the magnetic field strength at the exact time and location of the event, whereas the NW/IN mask shown in Sect. 3.2 (cf. Wedemeyer et al. 2020) is only providing a first reference for the overall properties of the magnetic environment. In summary, the lowered occurrence of events at times and locations of high magnetic field strength is in line with the observed signatures likely being caused by propagating shock waves.

\subsection{Identification of shock waves.}

Based on the analysis of the mm-wavelength observables from the 1D numerical models (Sect. 4.1), we adopt here the following criteria for shock wave signatures.

(i) A brightness temperature excess, $\Delta T_{\mathrm{b}}$, on order of a few hundred to thousands of Kelvin.

(ii) Lifetime on the order of tens of seconds.

(iii) Small lateral motion with speeds of less than a few tens of kilometres per second.

(iv) Low magnetic field strength.

Taking a closer look at the selected events A-D in Sect. 3.4 reveals that they fulfill all of the conditions in the list above. The events show significantly large brightness temperature excess $(575-1150 \mathrm{~K})$ as well as satisfying lifetimes (43-77 s) (Figs. 8-B.2). As indicated in Sect. 4.1, a shock wave propagates through the chromosphere with typical vertical speed on the order of $\sim 10 \mathrm{~km} \mathrm{~s}^{-1}$ and only a relatively small apparent motion within a few tens of kilometres per second is therefore expected. Events A-C show apparent speeds of between $\sim 12$ and $22 \mathrm{~km} \mathrm{~s}^{-1}$, while event D appears horizontally stationary (Figs. 7 and 8-B.2). All of the events (A-D) show velocities that lie within the regime expected for shock waves. Finally, all of the locations of the events only show a very small absolute magnetic field strength (Fig. 4). The fulfillment of the above criteria by the events in the ALMA observations do suggest that their signatures could originate from shock wave events.

The evolution of the surroundings of an event can provide clues as to the local atmospheric structure. At the position of event A, the brightness temperature increases and decreases repetitively independent of the surroundings (Fig. 8), which could be interpreted as a case of a wave train with multiple shock waves being excited at the same location in the photosphere below (see, e.g., Wedemeyer et al. 2004). In event D, it is possible to see the indications of a repetitive shock wave pattern at the center location (Fig. B.2h). Although, the previous $T_{\mathrm{b}}$ peaks are relatively weak.

The low spatial resolution makes it challenging to estimate motions on the short timescales of the lifetimes of the events. Further, the apparent motion of an event might be difficult to estimate if the event not well isolated. The low apparent motion 

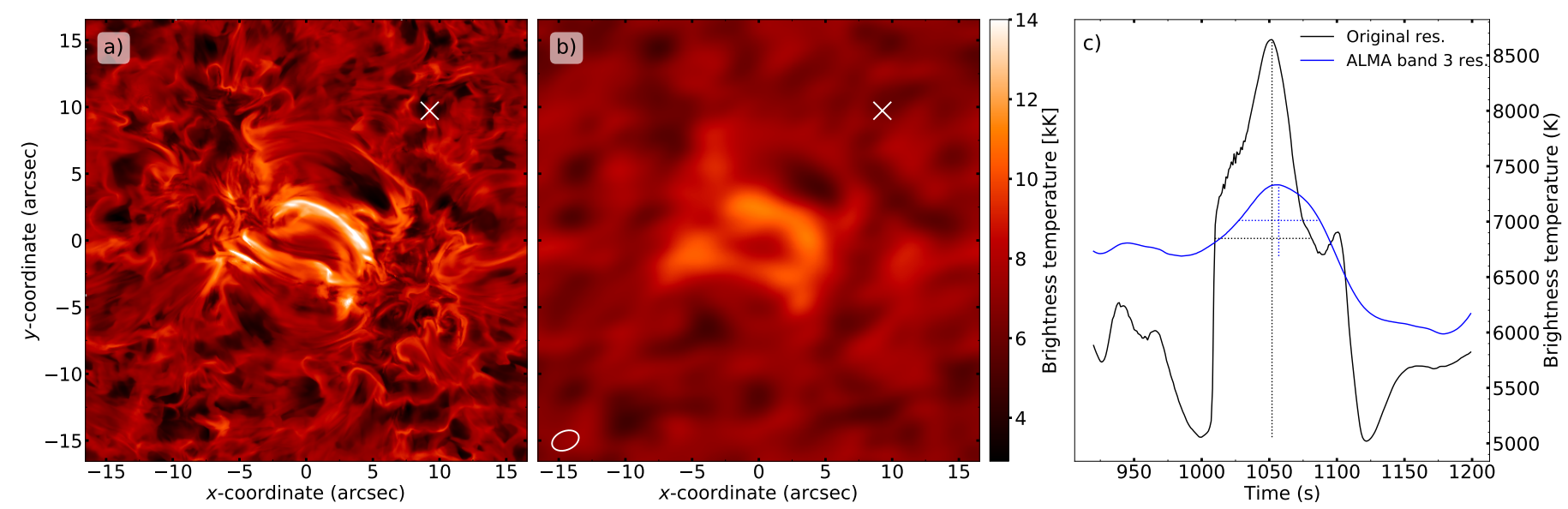

Fig. 12. Example of degradation with lower spatial resolution of shock wave $T_{b}$ signature from the 3D Bifrost numerical model. (a) Synthetic brightness temperature map with a cell size of $\sim 0.066^{\prime \prime}$ averaged over the frequencies of ALMA band 3 at $t=1052 \mathrm{~s}$. The white cross marks the location of the selected event, $(x, y)=(9.26,9.72) \mathrm{Mm}$. $(b)$ The synthetic brightness temperature map degraded to the spatial resolution of the ALMA band 3 observations by convolution with the clean beam of observational data. The FWHM of the clean beam is marked by the white ellipse for reference. $(c)$ Time evolution of the brightness temperature of the original and convolved maps at the selected location between $t=920 \mathrm{~s}$ and $1200 \mathrm{~s}$. The vertical and horizontal dotted lines mark the $T_{\mathrm{b}}$ excess amplitude and lifetime of the shock wave signature in the respective resolution.

of event D could possibly be explained by the fact that it is unresolved. Nevertheless, the significant apparent motion spanning several pixels of the brightest points in examples A-C over their lifetimes brings reliability to the speed estimates.

The approach of looking for variations in the brightness temperature at a fixed location (time evolution for each pixel) gives, in principle, indications of all kinds of dynamical small-scale features. Out of the 552 registered events, it seems that most of the events exhibit an increase and decrease in brightness temperature at a more or less fixed (horizontal) location (see Figs. 2 and 7), as expected for a mostly vertically propagating shock wave. The peaks in the pixels associated with an individual event occur very close in time, separated by no more than a few seconds, indicating that the peaks are caused by the same underlying physical event. The majority of events show a speed of apparent motion very close to zero which could probably be a result of low spatial resolution with under-resolved events and a slight shift towards higher velocities could be expected with an increase in spatial resolution.

Many of the 552 detected events (Figs. 5-7) fulfill the aforementioned criteria which might suggest they do originate from shock waves. As a direct result of the selection process of the events, all of them show a high variation in brightness temperature. Approximately $95 \%$ of the events show an absolute LOS magnetic field strength lower than $20 \mathrm{G}$ and an apparent speed of less than $30 \mathrm{~km} \mathrm{~s}^{-1}$. Further, as discussed in Sect. 4.3 , it is very plausible that the lifetimes indicated by the 1D simulations (Sect. 4.1) are underestimated and that longer lifetimes would be expected in the observations. Assuming that lifetimes could be as long as $100 \mathrm{~s}$ would result in more than half of all detected events potentially being produced by shock waves. However, further studies are required using more realistic numerical models to determine to what degree the lifetimes are underestimated.

\subsection{Numerical simulations: $1 D$ versus $3 D$}

The shape of the temporal $T_{\mathrm{b}}$ profile of a shock wave signature in a $3 \mathrm{D}$ atmosphere often deviates significantly from the characteristic shape that is indicated in the 1D simulations. The shock wave is propagating through an inhomogenous 3D atmosphere, where disturbances from previous dynamics have made an imprint or the shock wave might be interfered by other simultaneous dynamics. Spatial smearing, as well as severely changing the amplitude of the $T_{\mathrm{b}}$ peak, might also considerably deform the temporal profile (see Fig. 12). The temporal profile is therefore highly dependent on the surrounding structure but can at least be used to validate clear cases. The numerical RADYN simulations in this study provide important insight into the expected signatures of shock waves as observed at $\mathrm{mm}$ wavelengths with ALMA. On the other hand, the restriction to one spatial dimension is detrimental to investigations in view of the complex three-dimensional structure of the solar atmosphere. The one-dimensional numerical models therefore only provide a first approximation of the radiative signatures of a propagating shock wave.

A more extensive study of 2D brightness temperature maps from numerical 3D simulations would provide a more realistic estimate and indication of the appearance of shock wave signatures at mm wavelengths as seen by ALMA. With these, the complexity of the 3D atmosphere would be accounted for. Also, the degree of degradation of dynamical fine structure that comes with limited spatial resolution can be determined, which by nature is not possible with a one-dimensional approach.

\section{Conclusions and outlook}

Analyzing an ALMA Band $3(\sim 3 \mathrm{~mm})$ observation of a diskcenter quiet Sun region, we detected 552 small-scale dynamic events. An extensive study of the physical parameters of all events was carried out in order to characterise the events and reveal statistical trends. The possibility that the detected signatures come from shock wave events was examined. For that purpose, we used predictions of observable shock waves signatures at $\mathrm{mm}$ wavelengths from one-dimensional atmospheric models. The detected events show a large spread in brightness temperature excess $\left(\Delta T_{\mathrm{b}}\right)$ reaching up to $\sim 1200 \mathrm{~K}$ for some very strong events and lifetimes of between $\sim 43$ and $360 \mathrm{~s}$. The typical values, represented by approximately half of the events, are $\sim 450-750 \mathrm{~K}$ and $\sim 55-125 \mathrm{~s}$, respectively. However, most of the 
events show a $T_{\mathrm{b}}$ excess that is smaller than predicted by the $1 \mathrm{D}$ simulations.

We conclude that the restriction to one spatial dimension is a severe limitation that cannot properly account for the smallscale chromospheric pattern as a product of complex 3D wave propagation. Observations with the limited spatial resolution of ALMA Band 3 are therefore subject to spatial smearing of the pattern, resulting in a strong reduction of the observable brightness temperature excess $\left(\Delta T_{\mathrm{b}}\right)$.

Simulations of 3D shock wave propagation shown here and in previous studies (Wedemeyer-Böhm et al. 2007; Loukitcheva et al. 2015; Wedemeyer et al. 2020) demonstrate that the brightness temperature variations at $\mathrm{mm}$ wavelengths are strongly reduced as a result of limited spatial resolution as currently achieved with ALMA in Band 3. The magnitude of the dynamic $T_{\mathrm{b}}$ events seen in the observations could be considered as a lower limit. Based on these results, it is likely that the observed parameters are in line with the values predicted by the simulations when properly accounting for the dynamic 3D structure of the solar chromosphere and the effect of limited spatial resolution during the observation. Taking this into consideration, it seems plausible that many of the detected events have signatures originating in propagating shock waves. A more detailed study based on 3D numerical simulations is needed and will be presented in a forthcoming paper.

Acknowledgements. This work is supported by the SolarALMA project, which has received funding from the European Research Council (ERC) under the European Union's Horizon 2020 research and innovation programme (grant agreement No. 682462) and by the Research Council of Norway through its Centres of Excellence scheme, project number 262622. This paper makes use of the following ALMA data: ADS/JAO.ALMA\#2016.1.00423.S. ALMA is a partnership of ESO (representing its member states), NSF (USA) and NINS (Japan), together with NRC(Canada), MOST and ASIAA (Taiwan), and KASI (Republic of Korea), in co-operation with the Republic of Chile. The Joint ALMA Observatory is operated by ESO, AUI/NRAO and NAOJ. We are grateful to the many colleagues who contributed to developing the solar observing modes for ALMA and for support from the ALMA Regional Centres. We also acknowledge collaboration with the Solar Simulations for the Atacama Large Millimeter Observatory Network (SSALMON, http: //www. ssalmon.uio.no).

\section{References}

Alissandrakis, C. E., Patsourakos, S., Nindos, A., \& Bastian, T. S. 2017, A\&A, 605, A78

Carlsson, M., \& Stein, R. F. 1992, ApJ, 397, L59
Carlsson, M., \& Stein, R. F. 1994, in Chromospheric Dynamics, ed. M. Carlsson, 47

Carlsson, M., \& Stein, R. F. 1997, ApJ, 481, 500

Carlsson, M., \& Stein, R. F. 2002, ApJ, 572, 626

Carlsson, M., Hansteen, V. H., Gudiksen, B. V., Leenaarts, J., \& De Pontieu, B. 2016, A\&A, 585, A4

Carlsson, M., De Pontieu, B., \& Hansteen, V. H. 2019, ARA\&A, 57, 189

Cornwell, T. J. 2008, IEEE J. Sel. Top. Sign. Process., 2, 793

de la Cruz Rodríguez, J., \& van Noort, M. 2017, Space Sci. Rev., 210, 109

de Wijn, A. G., Stenflo, J. O., Solanki, S. K., \& Tsuneta, S. 2009, Space Sci. Rev., 144, 275

Dorfi, E. A., \& Drury, L. O. 1987, J. Comput. Phys., 69, 175

Eklund, H., Wedemeyer, S., Snow, B., et al. 2020, Phil. Trans. A R. Soc., 379, 20200185

Everitt, B. 1972, Br. J. Psychiatry, 120, 143

Gafeira, R., Jafarzadeh, S., Solanki, S. K., et al. 2017, ApJS, 229, 7

Gudiksen, B. V., Carlsson, M., Hansteen, V. H., et al. 2011, A\&A, 531, A154

Guevara Gómez, J. C., Jafarzadeh, S., Wedemeyer, S., et al. 2020, Phil. Trans. R. Soc. A., 379, 20200184

Jafarzadeh, S., Solanki, S. K., Gafeira, R., et al. 2017a, ApJS, 229, 9

Jafarzadeh, S., Solanki, S. K., Stangalini, M., et al. 2017b, ApJS, 229, 10

Jafarzadeh, S., Wedemeyer, S., Szydlarski, M., et al. 2019, A\&A, 622, A150

Jafarzadeh, S., Wedemeyer, S., Fleck, B., et al. 2020, Phil. Trans. R. Soc. A, in press [arXiv:2010.01918]

Lemen, J. R., Title, A. M., Akin, D. J., et al. 2012, Sol. Phys., 275, 17

Loukitcheva, M., Solanki, S. K., Carlsson, M., \& Stein, R. F. 2004, A\&A, 419 747

Loukitcheva, M., Solanki, S. K., \& White, S. 2006, A\&A, 456, 713

Loukitcheva, M., Solanki, S. K., \& White, S. M. 2009, A\&A, 497, 273

Loukitcheva, M., Solanki, S. K., Carlsson, M., \& White, S. M. 2015, A\&A, 575, A15

Loukitcheva, M. A., White, S. M., \& Solanki, S. K. 2019, ApJ, 877, L26

Morton, R. J. 2012, A\&A, 543, A6

Nindos, A., Alissandrakis, C. E., Bastian, T. S., et al. 2018, A\&A, 619, L6

Okamoto, T. J., \& De Pontieu, B. 2011, ApJ, 736, L24

Patsourakos, S., Alissandrakis, C. E., Nindos, A., \& Bastian, T. S. 2020, A\&A, 634, A86

Pereira, T. M. D., \& Uitenbroek, H. 2015, A\&A, 574, A3

Pesnell, W. D., Thompson, B. J., \& Chamberlin, P. C. 2012, Sol. Phys., 275, 3

Rodger, A. S., Labrosse, N., Wedemeyer, S., et al. 2019, ApJ, 875, 163

Ryle, M. 1975, Science, 188, 1071

Schou, J., Scherrer, P. H., Bush, R. I., et al. 2012, Sol. Phys., 275, 229

Uitenbroek, H. 2001, ApJ, 557, 389

Vernazza, J. E., Avrett, E. H., \& Loeser, R. 1981, ApJS, 45, 635

Wedemeyer, S., Freytag, B., Steffen, M., Ludwig, H. G., \& Holweger, H. 2004, A\&A, 414, 1121

Wedemeyer, S., Bastian, T., Brajša, R., et al. 2016, Space Sci. Rev., 200, 1

Wedemeyer, S., Szydlarski, M., Jafarzadeh, S., et al. 2020, A\&A, 635, A71

Wedemeyer-Böhm, S., Ludwig, H. G., Steffen, M., Leenaarts, J., \& Freytag, B. 2007, A\&A, 471, 977

White, S. M., Iwai, K., Phillips, N. M., et al. 2017, Sol. Phys., 292, 88

Wilson, T. L., Rohlfs, K., \& Hüttemeister, S. 2013, Tools of Radio Astronomy, Astron. Astrophys. Lib. (Springer-Verlag Berlin Heidelberg)

Wöger, F., Wedemeyer-Böhm, S., Schmidt, W., \& von der Lühe, O. 2006, A\&A, 459, L9 
H. Eklund et al.: The Sun at millimeter wavelengths. II.

\section{Appendix A: Example of a shock wave in a Bifrost 3D simulation}

Figure A.1 shows the evolution of the gas temperature in a small region of the solar atmosphere as part of a 3D magneto-hydrodynamical simulation with the Bifrost code
(Gudiksen et al. 2011; Carlsson et al. 2016). The displayed data exhibit an example of shock wave propagation. This example is used to illustrate the connection between the atmospheric thermal structure and the observable brightness temperature in mm-wavelength radiation maps as discussed in Sect. 4.2.

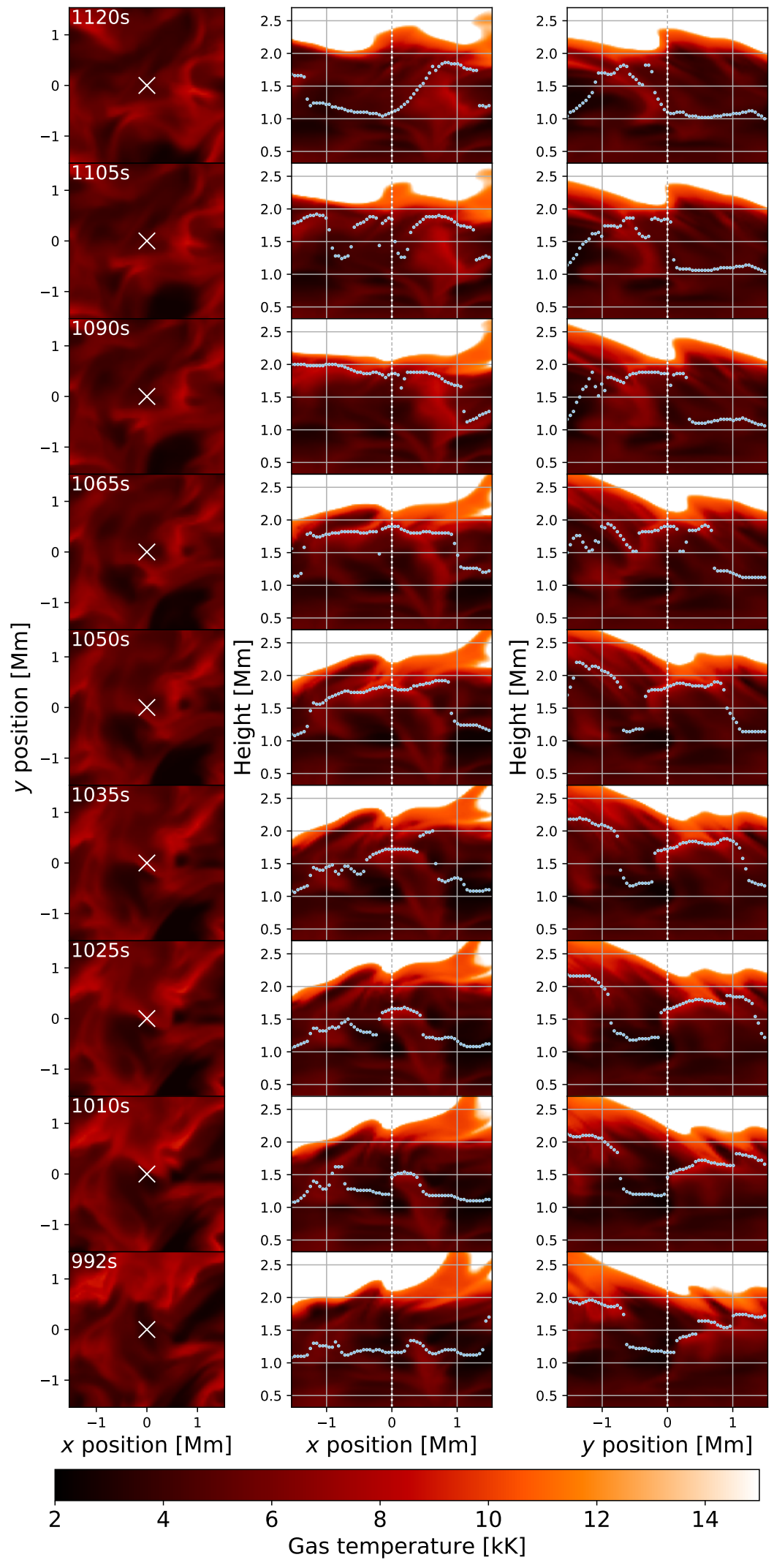

Fig. A.1. Illustration of a propagating shock wave in the Bifrost 3D simulation. Gas temperature surrounding the shock wave example is shown at nine time steps between $t=992 \mathrm{~s}$ and $t=1120 \mathrm{~s}$ of the simulation run. The leftmost column shows horizontal cuts (top-view) at a height of $z=1.5 \mathrm{Mm}$, and the middle and rightmost columns show vertical cuts with the height versus the $x$ and $y$-coordinates, respectively. The blue markers show the formation height of unity optical depth $\tau=1.0$ for the mm wavelenghts of ALMA band $3(2.78-3.26 \mathrm{~mm})$. The white crosses and the vertical white dashed line at $(x, y)=(0,0)$ mark the location of the sampled $T_{\mathrm{b}}$ signature in Fig. 12c. At $t=992 \mathrm{~s}$ the shock front is visible and the mm-wavelength intensities at $\tau=1.0$ are coupled with it at $(x, y, z)=(0.3,0.3,1.2) \mathrm{Mm}$. The front is propagating mostly upwards and inwards towards $(x, y, z)=(0,0,1.6) \mathrm{Mm}$ at $t=1025 \mathrm{~s}$. The $T_{\mathrm{b}}$ signature is peaking in magnitude at this location around $t=1050 \mathrm{~s}$, when a cooler post-shock region is visible where the front has passed. The mm-wavelength intensities are tracking the shock front upwards to $z \approx 2.0 \mathrm{Mm}$ around $t=1090 \mathrm{~s}$ and thereafter decouple and sample the post-shock region $(t=1120 \mathrm{~s})$. See Eklund et al. (2020) for a more detailed analysis of propagating shock waves in the Bifrost simulation. 


\section{Appendix B: Detailed study of additional examples of brightness temperature events in the ALMA data}

An additional couple of examples of $T_{\mathrm{b}}$ events found in the observational data are shown in detail. These events are marked as " $B$ " and " $D$ " in the figures in the main text.

Event B (see Fig. B.1) is located slightly closer to the magnetically stronger areas in the upper FOV at $(x, y)=$ $\left(-0.2^{\prime \prime}, 3.4^{\prime \prime}\right)$, precisely on the border of the NW mask, though the absolute magnetic field strength is low at about $56 \mathrm{G}$. Event B shows a similar evolution in brightness temperature to event A. There is a rise in $T_{\mathrm{b}}$ from about $6890 \mathrm{~K}$ to $7960 \mathrm{~K}$ in $90 \mathrm{~s}$ followed by a decrease to about $6910 \mathrm{~K}$ over the course of $100 \mathrm{~s}$. Event B has therefore a brightness temperature excess of
$\Delta T_{\mathrm{b}}=1055 \mathrm{~K}$ and a lifetime of $77 \mathrm{~s}$. The profile of the temporal evolution of the brightness temperature (Fig. B.1h) shows a very similar shape to the fiducial run of the one-dimensional simulations. Event B, with a size of $\sim 3^{\prime \prime}$ at the temperature of FWHM of the main peak, shows an apparent motion of approximately $12 \mathrm{~km} \mathrm{~s}^{-1}$.

Event D (Fig. B.2) at a location of $(x, y)=\left(-11.0^{\prime \prime},-5.6^{\prime \prime}\right)$ shows a rapid increase in brightness temperature from $6585 \mathrm{~K}$ to $7160 \mathrm{~K}$ followed by a decrease to $6450 \mathrm{~K}$. The resulting amplitude and lifetime are $\Delta T_{\mathrm{b}}=575 \mathrm{~K}$ and $65 \mathrm{~s}$, respectively. The event appears horizontally stationary without any strong indications of apparent motion. At the specific location there are hints of previous peaks of brightness temperature excess (Fig. B.2h), merely $200-300 \mathrm{~K}$ approximately $6 \mathrm{~min}$ and $3.5 \mathrm{~min}$ before event D.

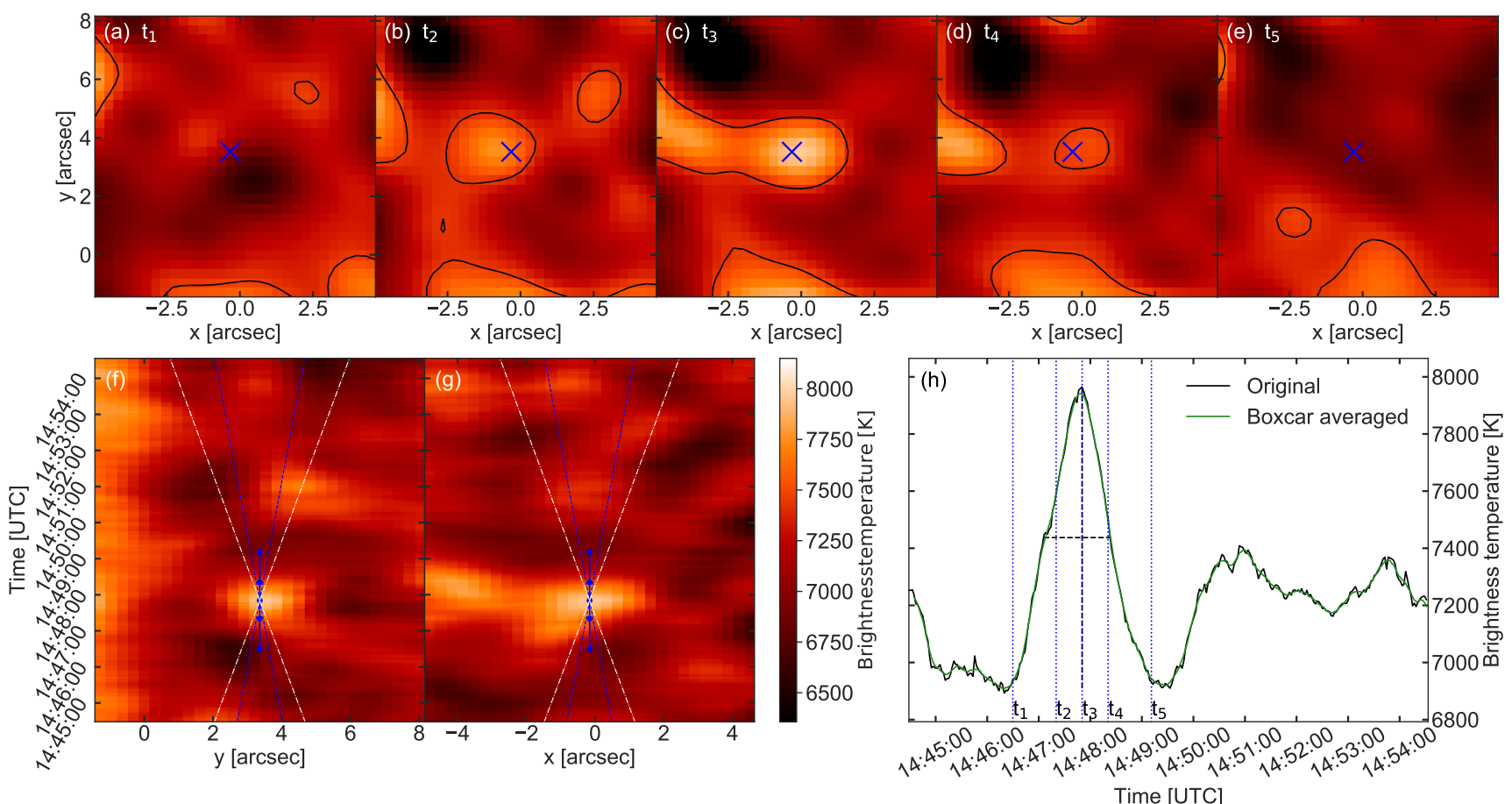

Fig. B.1. Detailed study of event B. (a)-(e) Close-ups of the surrounding brightness temperatures at different time steps, $t_{1}$ to $t_{5}$ from left to right, through the shock wave event. The time of the $T_{\mathrm{b}}$ peak is marked by $t_{3}=14: 47: 50$. Here, $t_{1}, t_{2}, t_{4}$ and $t_{5}$ mark $-80,-30,30$, and $80 \mathrm{~s}$ from the peak, respectively. The contour lines mark the half maximum of the maximum $\Delta T_{\mathrm{b}}$ peak and the blue crosses mark the center location. $(f)-(g)$ Space-time diagrams for a vertical and horizontal slit across the FOV at the center location. The center location for time steps $t_{1}$ to $t_{5}$ is marked with blue dots connected with a line. Velocity slopes for 10 and $20 \mathrm{~km} \mathrm{~s}^{-1}$ are indicated by blue dotted and white dashed lines, respectively. $(h)$ The time evolution of the brightness temperature of the center location, where the time steps $t_{1}$ to $t_{5}$ are indicated by blue dotted lines. Both the original data (black) and the averaged data (green) are shown. The horizontal and vertical dashed lines mark the event lifetime and brightness temperature excess, respectively. 
H. Eklund et al.: The Sun at millimeter wavelengths. II.
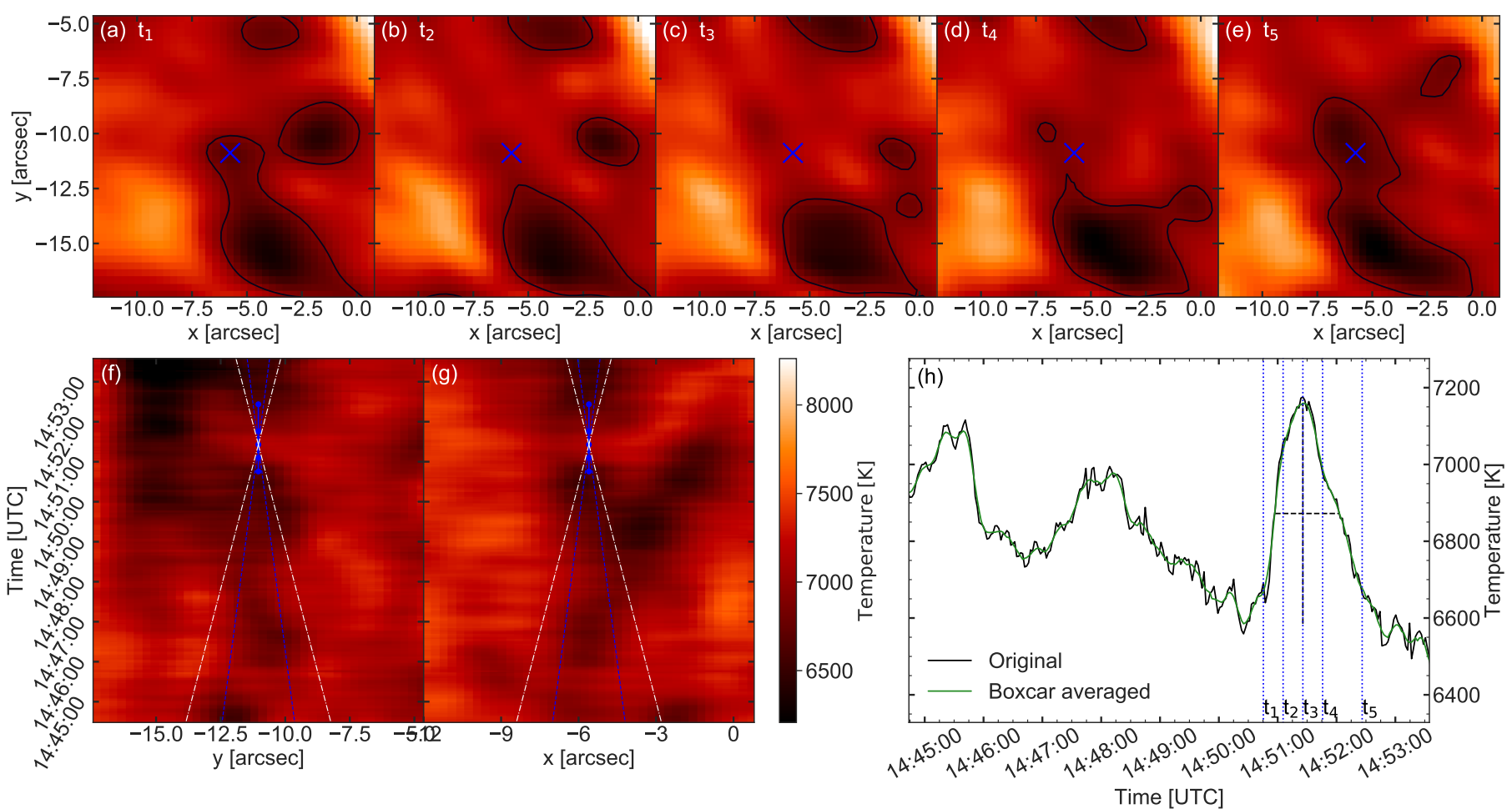

Fig. B.2. Detailed study of event D, as for event B in Fig. B.1. The time of the $T_{\mathrm{b}}$ peak is marked by $t_{3}=14: 51: 25 . t_{1}, t_{2}, t_{4}$ and $t_{5}$ mark $-40,-20,20$ and $40 \mathrm{~s}$ from the peak, respectively. 